\title{
LA RÉPARATION INDIVIDUELLE EN APPLICATION DES MÉCANISMES PRÉVUS PAR LE DROIT INTERNATIONAL HUMANITAIRE
}

\section{Sophie Rondeau*}

Le présent article fait état d'un questionnement sur l'état actuel du rôle des normes juridiques émanant du système de droit international humanitaire (DIH) en ce qui a trait au droit à la réparation, en prenant soin de mettre la personne en tant que victime de la guerre au centre de notre réflexion. En considérant la notion de réparation sous l'angle de la victime comme un tout à décrire et à analyser, nous cherchons à savoir sỉl existe un droit à la réparation que possède la victime d'un conflit armé régi par le droit international humanitaire. Le fondement même de cette recherche sappuie donc sur le cadre normatif conventionnel du DIH régissant la notion de réparation, que cette dernière accorde ou non un droit à une victime.

This paper presents a series of questions on the present state of the role of judicial standards arising from the system of international humanitarian law [IHL] as regards the right to compensation, by making it a point to place the person as a war victim at the center of our reflection. In considering the concept of compensation from the angle of the victim as a whole, we seek to know whether there exists a right to compensation to which the victim of an armed conflict governed by international bumanitarian law is entitled. The very foundation of this research is thus based on the conventional normative framework of IHL governing the concept of compensation, whether or not it grants a right to a victim.

\section{INTRODUCTION: LA JUSTICIABILITÉ DES DROITS HUMANITAIRES ET LA RESPONSABILITÉ INDIVIDUELLE DES OBLIGATIONS HUMANITAIRES}

Le droit international humanitaire ${ }^{1}$ illustre comment le système juridique international est de moins en moins confiné uniquement aux relations interétatiques, évoluant ainsi au-delà de son orthodoxie juridique traditionnelle, celle-là même qui s'inscrit dans la droite ligne de la conception westphalienne de l'ordre mondial. S'il n'y avait donc pas, originellement, de rapport d'assujettissement

\footnotetext{
* LL.B., LL.M. (droit international) (Université du Québec à Montréal); Membre du Barreau du Québec. Ce texte est extrait de : S. Rondeau, Violations du droit international humanitaire et réparation : la place individuelle de la victime, thèse de maîtrise en droit international (LL.M.), Université du Québec à Montréal, mars 2008 [non publié].

1 À noter que l'utilisation, dans le présent article, des expressions " droit international humanitaire ", « droit humanitaire " et " DIH » réferent toutes à la même notion.
} 
entre les deux strates formant le droit international public (la première correspondant à une couche traditionnelle étatique reposant sur l'autre, formée par le "droit constitutionnel et administratif de la communauté internationale $»^{2}$ ) nous voyons aujourd'hui les six milliards d'êtres humains constituant la population des États se forger une place dans ce système juridique. Le droit international humanitaire - ce droit régissant la guerre, phénomène considéré comme le fondement ontologique de l'État ${ }^{3}$ - est à la fois le témoin et l'instigateur de cette évolution.

En effet, comme l'a affirmé Jean Pictet au sujet de la place éminente que tient la Convention de Genève pour l'amélioration du sort des militaires blessés dans les armées en campagne du 22 août 1864 dans l'évolution du droit des gens : " pour la première fois, [...], une réglementation internationale est centrée, non plus sur des intérêts étatiques, mais sur la seule protection de l'individu ${ }^{4}$. On ne doit toutefois pas se borner au sens littéral des mots et voir dans une telle affirmation une consécration pure et simple de droits justiciables humanitaires individuels pour les victimes de conflits armés. Rappelons-nous, tout d'abord, qu'en matière de protections édictées par le droit humanitaire, il est question de catégories de personnes, et non d'individus. Aussi, dans la vision traditionnelle du DIH (qui existait avant l'émergence des droits humains), on peut interpréter l'expression " protection de l'individu » utilisée par Pictet comme signifiant une forme de protection d'un intérêt objectif de l'État, ce dernier n'existant en réalité qu'à travers ses citoyens envisagés de manière individuelle.

S'il est vrai que le DIH édicte des protections bénéficiant aux individus (ou, tout au moins, aux groupes d'individus s) affectés par les conflits armés, la façon dont s'articule la mise en oeuvre post bellum de ces protections en cas de viola-

2 Cette illustration du droit international en deux couches reprend le concept expliqué dans l'ouvrage : Marco Sassòli et Antoine Bouvier, Un droit dans la guerre?, Genève, Comité international de la Croix-Rouge, 2003, aux pp. 92 et 93 [ci-après Sassòli Bouvier].

3 Philip S. Golub, "Cinq années de "guerre au terrorisme" - État d'urgence permanent " $L e$ Monde diplomatique (septembre 2006) aux pp. 18 et 19, en ligne : Le monde diplomatique $<$ http://www.monde-diplomatique.fr/2006/09/GOLUB/13920> (date d'accès : $1^{\text {er }}$ décembre 2009).

4 O. Uhler, H. Coursier et al., "Commentaire de la Convention (IV) de Genève relative à la protection des personnes civiles en temps de guerre du 12 août 1949 ", dans J. Pictet, dir., Commentaires des Conventions de Genève du 12 aồt 1949 . Volume IV, Genève, Comité international de la Croix-Rouge, 1956, art. 8, à la p. 84 [ci-après Commentaire de la Convention $I V$., en ligne : CICR, droit international humanitaire <http://www.icrc.org/dih.nsf/COM/380600011?OpenDocument> (date d'accès : $1^{\text {er }}$ décembre 2009) [ci-après Commentaires du CICR CGIV]. Voir aussi : George Abi-Saab, "The Specificities of Humanitarian Law " dans Christophe Swinarski, dir., Études et essais sur le droit international humanitaire et sur les principes de la Croix-Rouge en l'honneur de Jean Pictet / Studies and Essays of International Humanitarian Law and Red Cross Principles in Honour of Jean Pictet, Genève et La Haye, Comité international de la Croix-Rouge,, 1984, aux pp. 265, 269 : « [ t] he thrust of the Conventions (and Protocols) is to go beyond the interstate level and to reach for the level of the real (or ultimate) beneficiaries of humanitarian protection, i.e. individuals and groups of individuals ".

5 En droit international humanitaire, la terminologie differe, selon que le conflit visé soit international ou non international. Par exemple, il est question de " personnes qui participent directement aux hostilités » lorsqu'il est question de conflit armé non international, tandis qu'il est question de " combattants " et de "prisonniers de guerre " lorsqu'on se trouve devant un conflit armé international. 
tions semble moins évidente à définir. Les personnes qui sont protégées par le DIH détiennent-elles des droits à proprement parler? Car, force est de constater qu'actuellement, le DIH ne prévoit pas de mécanisme judiciaire spécifique dont une victime pourrait se prévaloir pour déterminer la réparation qui lui est due lorsqu'elle est victime d'une violation du droit humanitaire.

En effet, un des défis constants du droit international public, et a fortiori du droit humanitaire, réside dans le respect de son application. Dans un contexte de conflit armé, les mécanismes a priori (principalement la diffusion et la formation en temps de paix) sont habituellement privilégiés. Cela ne veut toutefois pas dire que la mise en ouvre a posteriori est totalement évacuée. La sanction judiciaire des violations, par le truchement de la responsabilité internationale de l'État et la responsabilité pénale de l'individu constitue en effet un moyen de mise en oeuvre du DIH.

Cependant, ces deux mécanismes connaissent leurs des limites : tout d'abord, la responsabilité étatique telle qu'on l'entend au sens classique du droit international public général ${ }^{6}$ et qui est le fondement de la responsabilité des États en $\mathrm{DIH}$, consacre bel et bien la règle de base de la réparation du préjudice subi à la suite d'une violation d'un droit? Il faut néanmoins comprendre que ce principe s'applique aux relations interétatiques et qu'il n'est pas question, ici tout au moins, de faire entrer un individu dans l'équation ${ }^{8}$. Ceci explique donc en grande partie pourquoi les réclamations en réparation en faveur des individus se sont traditionnellement réalisées à travers le mécanisme de la protection diplomatique'. En ce qui a trait aux poursuites pénales, il est vrai qu'elles constituent

6 Voir, par ex., le Projet d'articles sur la responsabilité de l'État pour fait internationalement illicite dans Rapport de la Commission de droit international, Doc. Off. AG NU, 53 esess., supp. $\mathrm{n}^{\circ}$ 10, Doc. NU A/56/10 (2001) 45, art. 31, al. 1[ci-après Projet d'articles sur la responsabilité de l'État] et Annexe de la Charte des Nations Unies : Statut de la Cour internationale de Justice, , 26 juin 1945,, R.T. Can.1945 no 7, art. 36, al. 2. Voir aussi Commentaries to the draft articles on Responsibility of States for internationally wrongful acts, adopted by the International Law Commission at its fifty-third session, Doc. Off. AG NU, 56 esess., supp. $\mathrm{n}^{\circ} 10$ Doc. NU A/56/10 (2001), chap.IV.E.2, à la p. 234 et s.

7 " [C]'est un principe du droit international, voire une conception générale du droit, que toute violation d'un engagement comporte l'obligation de réparer. [...] La réparation est le complément indispensable d'un manquement à l'application sans qu'il soit nécessaire que cela soit inscrit dans la convention même. » : Usine de Chorzòw (Allemagne c. Pologne) (1928), C.P.J.I. (sér. A/B) no 17 à la p. 29.

8 " [I]n inter-state relations, whether States claims are made on behalf of a State's national or on behalf of the State itself, they are always claims of the State ": Barcelona Traction, Light and Power Company, Limited (Belgique c. Espagne), [1970] C.I.J. rec. 3 à la p. 46. Voir aussi Rainer Hofmann and Frank Riemann, Compensation for victims of war - Background Report, International Law Association, Committee on Compensation for Victims of War, 17 mars 2004, pour d'autres décisions où l'État réclame compensation pour des dommages subis par lui et ses ressortissants, en ligne: International Law Association, Committee on Compensation for Victims of War, « Download Background Report » <http://www.ila-hq.org/en/committees/ index.cfm/cid/1018> (date d'accès: $1^{\text {er }}$ décembre 2009).

9 La théorie de la protection diplomatique telle que définit par le Professeur Rousseau consiste en "l'action diplomatique entreprise par le gouvernement du particulier lésé auprès du gouvernement présumé responsable pour obtenir la réparation du dommage causé à son ressortissant »: Charles Rousseau, Droit international public, t. 5, 7e éd., Paris, Sirey, 1973 à la p. 97. Voir aussi, pour une étude approfondie de la théorie de la protection diplomatique, Bertrand 
un maillon important de la mise en œuvre, mais elles ne devraient pas décourager les efforts visant à prévenir les violations et protéger les victimes par des moyens autres ${ }^{10}$.

Il est donc à-propos d'examiner les fondements juridiques du droit à la réparation en DIH sous l'angle des droits primaires et des droits secondaires pour s'attarder, ensuite, à l'analyse de l'application directe du DIH en droit interne, via l'examen des jurisprudences nationales dans lesquelles des recours individuels en réparation contre l'État ont été présentés. À la suite de cet examen, nous terminerons notre réflexion en regardant brièvement l'opportunité et la viabilité d'un mécanisme international de plainte qui serait accessible aux victimes de violations du DIH.

\section{LES FONDEMENTS JURIDIQUES DU DROIT À LA RÉPARATION EN DROIT INTERNATIONAL HUMANITAIRE}

La notion de réparation existe en $\mathrm{DIH}$, en relation avec la notion de répression. Lorsque la prévention et l'action n'ont pas eu les effets escomptés, les États parties aux Conventions de Genève ont l'obligation de faire cesser les infractions au droit humanitaire, de réprimer les infractions graves, mais aussi de payer des indemnités s'ils commettent, par l'intermédiaire des personnes faisant partie de leurs forces armées, ces violations. Cette obligation de réparation s'inscrit en premier lieu dans un élargissement des règles s'appliquant à la conduite des hostilités. Le raisonnement est le suivant : le droit limite les moyens et méthodes de combat, ce qui veut dire qu'il n'est pas possible pour les parties au conflit de faire la guerre de n'importe quelle façon. Si une partie au conflit va au-delà des limites prescrites par le droit, elles n'engagent pas uniquement sa responsabilité, elle se voit aussi forcée de remettre l'autre partie dans l'état où elle se trouverait avant que la violation ne soit commise. Par exemple, les États ayant dispersé des mines sans discrimination ou ayant causé d'autres atteintes illicites à l'environnement

Bauchot, La protection diplomatique des individus en droit international, mémoire de recherche, Université de Lille II, année scolaire 2001-2002 en ligne : <http://www.memoireonline.com/ link/603.html> (date d'accès : $1^{\text {er }}$ décembre 2009). Cette théorie repose donc sur l'hypothèse qu'un dommage causé à un ressortissant d'un État constitue de facto un dommage à l'État lui-même; c'est une application de la conception westphalienne du droit international et c'est la première manifestation du droit à la réparation au niveau international. Le fait que l'État puisse prendre fait et cause pour ses nationaux par la fiction de l'endossement lorsque ces derniers sont lésés par les autorités d'un autre État se justifie par le fait qu'un État à une obligation générale de protéger ceux-ci. De plus, en droit international public général, l'État a une compétence personnelle sur eux; en effet, le lien d'allégeance particulière qui est celui de la nationalité met en relation de subordination les ressortissants par rapport à leur État : Voir la section sur la compétence personnelle de l'État dans Nguyen Quoc Dinh, Patrick Daillier et Alain Pellet, Droit international public, 6e éd., Paris, LGDJ, 1999 à la p. 488 et s. À noter qu'en droit international humanitaire, la condition de nationalité n'est plus le principal motif d'allégeance. Voir la discussion sur le statut de victime en lien avec le concept de "personnes protégées » dans Procureur c. Tadi , (1999), Affaire n IT-94-1-A, aux para. 163-171 (Tribunal Pénal International pour l'ex-Yougoslavie, Chambre d'appel). et Procureur c. Delalic (1998), Affaire $n^{\circ}$ IT-96-21-T, aux para. 236 à 277 (Tribunal Pénal International pour l'ex-Yougoslavie, Chambre de $1^{\text {re }}$ instance).

10 Sassòli Bouvier, supra note 3 à la p. 304. 
sont tenus de payer une indemnité ${ }^{11}$. Malgré le fait que certaines règles générales applicables à la réparation en droit international public se voient modifiées lorsque le droit humanitaire est en cause ${ }^{12}$, on ne peut nier que le concept de réparation est intégré dans ce corpus juridique. On peut, malgré tout, se questionner sur sa raison d'être. En effet, l'imposition d'une obligation de réparation aux parties au conflit ne relèverait-t-elle pas, par exemple, d'une application du principe de réciprocité, plutôt que d'une volonté de remettre les victimes en état?

Dans la présente analyse, nous tenterons de voir quelle place le DIH réserve à l'individu en tant que victime, en regardant si les intérêts de cette dernière sont véritablement pris en compte et traduits sous forme de droits justiciables. Comme l'a affirmé Theodore Meron ${ }^{13}$, en dépit du fait que le droit de la guerre ait toujours contenu des règles visant à protéger des groupes de personnes (combattants et non-combattants), ce corpus juridique a été inévitablement animé par des considérations de stratégie militaire, de victoire et de réciprocité. Les sanctions, à l'instar des responsabilités, sont collectives : en temps de guerre, elles se matérialisent par les représailles des belligérants; après les hostilités, elles empruntent la forme de réparation de guerre. Dans ce contexte, la réparation en droit humanitaire peut-elle donc être aussi un droit individuel?

\section{A. Les droits primaires : existe-t-il des " droits humanitaires »?}

Le droit à la réparation est un droit secondaire, c'est-à-dire qu'il ne se concrétise que lorsqu'une série d'éléments préalables ont été réunis, y compris la contravention à une obligation prévue par le corps de la loi visé. Donc, pour savoir si les victimes de violations du DIH possèdent un droit de recours, dont un droit à réparation, il est primordial d'identifier, de prime abord, si des droits substantifs ont été attribués aux individus par le DIH.

Quoique le droit humanitaire ait été initialement formulé en termes de prohibitions s'adressant aux parties au conflit, un langage plus axé sur les droits s'est développé à travers les années. Dans les quatre Conventions de Genève de $1949^{14}$ et dans le Protocole additionnel aux Conventions de Genève du 12 août 1949 relatif

11 Voir "Rapport sur la protection des victimes de la guerre ", préparé par le Comité international de la Croix-Rouge pour la Conférence internationale pour la protection des victimes de la guerre, (1993) 803 Revue internationale de la Croix-Rouge 415.

12 Voir par ex. Sassòli Bouvier, supra note 3 aux pp. 297 et 298 : la responsabilité de l'État est absolue pour tous les actes commis par ses forces armées. Les représailles contre la population civile et les biens protégés, la réciprocité dans l'application des traités et la renonciation aux droits sont interdits.

13 Theodore Meron, "The Humanization of Humanitarian Law " (2000) 94 A.J.I.L. 239 aux pp. 242 et 243.

14 Respectivement : Convention (I) de Genève pour l'amélioration du sort des militaires blessés dans les armées en campagne, 12 août 1949, 75 R.T.N.U. 31 [ci-après CGI]; la Convention (II) de Genève pour l'amélioration du sort des blessés, malades et naufragés des forces armées sur mer, 12 août 1949, 75 R.T.N.U. 85 [ci-après CGII]; la Convention (III) de Genève relative au traitement des prisonniers de guerre, 12 août 1949, 75 R.T.N.U. 135 [ci-après CGIII]; la Convention (IV) de Genève relative à la protection des personnes civiles en temps de guerre, 12 août 1949, 75 R.T.N.U. 287 [ci-après CGIV ou la quatrième convention] Les textes de ces conventions sont disponibles en ligne : CICR <http://www.icrc.org/dih.nsf/CONVPRES?OpenView> (date d'accès : $1^{\text {er }}$ décembre 2009). 
à la protection des victimes des conflits armés internationaux du 8 juin $1977^{15}$ régissant les conflits armés internationaux, on répertorie près d'une quarantaine d'articles où le mot « droit " ${ }^{16}$ apparaît. Dans chacune de ces dispositions, les titulaires de ces droits sont les personnes protégées par chacune des conventions ${ }^{17}$. Que ce soit le droit du prisonnier de guerre de présenter aux autorités militaires qui le détiennent des requêtes concernant le régime de captivité auquel il est soumis ${ }^{18}$ ou le droit des personnes civiles protégées de s'adresser aux Puissances protectrices, au Comité international de la Croix-Rouge (CICR) ainsi qu'aux sociétés nationales ${ }^{19}$, il est clair que les individus, ou tout au moins, certaines catégories de personnes, possèdent des droits en vertu du régime du droit humanitaire applicable aux conflits armés internationaux. De plus, la clause d'inaliénabilité des droits que l'on retrouve dans les quatre Conventions de Genève dans laquelle on dispose que les personnes protégées « ne pourront en aucun cas renoncer partiellement ou totalement aux droits que leur assure la présente Convention ${ }^{20}$, vient reconnaître, de façon expresse, l'existence de ces droits.

Pour ce qui est des victimes des conflits armés non internationaux régis par l'article 3 commun aux Conventions de Genève et le Protocole additionnel visant la protection des victimes des conflits armés non internationau $x^{21}$, elles aussi possèdent des droits humanitaires. À notre avis, ces deux textes conferent aux personnes qui ne participent pas directement aux hostilités le droit d'être traitées avec humanité et sans distinction de caractère défavorable, le droit d'être protégées des atteintes portées à la vie, à l'intégrité corporelle et à la dignité ainsi que le droit de bénéficier des garanties judiciaires reconnues, même si le terme «droit » n'est pas utilisé expressément. Cette position fait écho aux propos tenus par Zegveld:

Apart from clear-cut examples of rules that can be conceptualized as "individual humanitarian rights", and with the purposes of IHL in mind, it is possible to identify many more rules that contain elements of individual benefits. For example, the grave breaches provisions could be construed as conferring individual humanitarian rights against acts such as willful killing, torture or inhuman treatment willfully causing great suffering or serious injury to body and health. The same holds true for norms applicable in non-international armed

151125 R.T.N.U. 3, art. 86 et 87 [ci-après PI].

16 Dans la version anglaise des conventions, le terme " entitled " est aussi utilisé pour référer au concept de "droit ". Voir par ex. CGIV, supra note 15, art. 27, en version anglaise : "Protected persons are entitled, in all circumstances, to respect for their person [...] ", en version française : «Les personnes protégées ont droit, en toutes circonstances, au respect de leur personne $[\ldots]$ ".

17 Voir de façon non exhaustive CGI et CGII, supra note 15, art. 6 et 7; CGIII, supra note 15, art. 6, 7, 14, 28(2), 54, 73, 81, 105, 106, 107 et 130; CGIV, supra note 15, art. 5, 7, 8, 27, 30, 35, $38,40,43,48,52(1), 71(2), 72,73,75(1), 76(6), 78(2), 80,101$ et 147; PI, supra note 16, art. $44(5), 45(3), 75$ et $85(4)$.

18 CGIII, supra note 15, art. 78.

19 Voir CGI, CGII et CGIII, supra note 15, art. commun 8 et 10 et CGIV, supra note 15, art. 9 et 11.

20 Supra note 15, art. 7/7/7/8 commun aux quatre Conventions de Genève.

211125 R.T.N.U. 609 [ ci-après PII]. 
conflicts, such as the prohibition of violence to life, outrages upon personal dignity, and humiliating and degrading treatment, stipulated in Article 3 common to the Geneva Conventions and in Article 4 of Additional Protocol II. ${ }^{22}$

Finalement, il faut noter que les « droits humanitaires » existent en faveur de toutes les victimes de conflits armés régis par le DIH, sans exception. Si les droits énumérés aux Conventions de Genève sont d'application universelle (ces textes ayant été ratifiés par l'ensemble des États), ceux prévus par leurs deux protocoles additionnels ne jouissent pas du même statut. En effet, environ deux tiers des États ont ratifié ces documents ${ }^{23}$. Toutefois, le caractère coutumier des normes conventionnelles attribuant des droits humanitaires qui sont contenues dans les Protocoles nous permet d'affirmer que ces droits existent dans tous les cas de conflits armés, peu importe que l'État impliqué ait ratifié ou non le protocole applicable au conflit dans lequel il est partie ${ }^{24}$.

\section{B. Les droits secondaires : Qu'est-ce que le droit à la réparation en vertu du DIH?}

Dans cette section, nous examinerons tout d'abord les sources primaires que constitue le droit positif contenu dans les traités de droit humanitaire au sujet de la réparation, pour ensuite nous pencher sur les sources secondaires que nous offre la doctrine relative à l'interprétation de ces dispositions.

Le droit applicable aux conflits armés internationaux consacre à l'article 3 de la Convention (IV) de La Haye concernant les lois et coutumes de la guerre sur terre du 18 octobre $1907^{25}$ et à l'article $91 P^{26}$ le droit d'obtenir réparation des préjudices subis à la suite de violations du $\mathrm{DIH}^{27}$. Ces dispositions font peser une

22 Liesbeth Zegveld, "Remedies for victims of violations of international humanitarian law " (2003) 851 Revue internationale de la Croix-Rouge 497 à la p. 504 [Zegveld, « Remedies for Victims "].

23 Le 30 juillet 2008, 169 États étaient parties au PI tandis que le 10 novembre 2009, 165 États étaient parties au PII.

24 Voir Jean-Marie Henckaerts et Louise Doswald-Beck, Customary International Humanitarian Law vol. 1 "Rules ", Genève et Cambridge, Comité international de la Croix-Rouge et Cambridge University Press, 2005, aux pp. 299-323. Le caractère coutumier des règles suivantes semble consacré, autant dans le cadre de conflits armés internationaux que non internationaux : l'obligation de traitement humain à la Règle 87 (aux pp. 306-308); l'interdiction de discrimination à la Règle 88 (aux pp. 308-311); la prohibition du meurtre à la Règle 89 (aux pp. 311-314); l'interdiction de la torture et de tout traitement inhumain à la Règle 90 (aux pp. 315319); l'interdiction du viol à la Règle 93 (aux pp. 323-327), etc. Pour prendre connaissance de l'état du droit conventionnel et de la pratique étatique (législation interne, manuels militaires, jurisprudence et déclarations officielles) ainsi que la pratique des organisations internationales et au sujet des règles coutumières du droit international humanitaire, voir le Vol. 2. [ci-après Étude du CICR sur le DIH coutumier].

2518 octobre 1907, 36 R.T.S.N. 539, art. 3 : « La Partie belligérante qui violerait les dispositions dudit Règlement sera tenue à indemnité, s'il y a lieu. Elle sera responsable de tous actes commis par les personnes faisant partie de sa force armée " [ci-après CIV (1907)].

26 Supra note 16, art. 91 : « La Partie au conflit qui violerait les dispositions des Conventions ou du présent Protocole sera tenue à indemnité, s'il y a lieu. Elle sera responsable de tous actes commis par les personnes faisant partie de ses forces armées ".

27 À noter que le Deuxième Protocole relatif à la Convention pour la protection des biens culturels en cas de conflit armé, 26 mars 1999, 38 I.L.M. 769, art. 38, mentionne lui aussi explicitement 
obligation de réparation aux États, en raison, entre autres, du champ d'application (les conflits armés internationaux) et de la formulation des deux articles précités qui reflète avec justesse les principes de base du droit international public.

Notons tout d'abord que la notion de réparation fait partie du droit international humanitaire coutumier. En effet, il est affirmé dans les Commentaires des Conventions de Genève du Comité International de la Croix-Rouge (CIRC) au sujet de l'article 91 PI que « cet article reproduit pour ainsi dire textuellement, sans l'abroger, ce qui signifie qu'il reste de droit coutumier pour tous, l'article 3 de la Convention de La Haye concernant les lois et coutumes de la guerre sur terre de $1907 »^{28}$. De plus, la Règle 150 contenue dans l'Étude du CICR sur le DIH coutumier de 2006 dispose qu'un État responsable pour les violations du droit international humanitaire doit effectuer une réparation totale pour la perte ou les blessures occasionnées, et ce, pour tout type de conflit armé2 29

En outre, on peut inférer l'obligation étatique de réparation des articles communs aux quatre Conventions de Genève traitant de la responsabilité des parties contractantes $^{30}$ : ces articles rappellent l'impossibilité d'exonération de responsabilité pour les Hautes Parties contractantes, lorsqu'il y a commission d'infractions graves. L'État demeure donc responsable des infractions aux Conventions, il ne peut refuser de reconnaitre sa responsabilité pour la raison que les auteurs ont été punis et il reste, par exemple, tenu de payer une indemnité ${ }^{31}$. En outre, lorsque ces articles communs aux quatre Conventions de Genève sont lus conjointement avec l'article $31 \mathrm{du}$ Projet d'article sur la responsabilité étatique pour fait internationalement illicite qui dispose que « l'État responsable est tenu de réparer intégralement le préjudice causé par le fait internationalement illicite ${ }^{32}$, on voit une consécration tacite de l'obligation de réparer le préjudice subi à la suite d'une violation des normes de droits humanitaires en temps de conflit armé international.

La notion de réparation existe donc en DIH, sans qu'il soit toutefois spécifié explicitement à qui cette obligation de réparation est due : est-ce uniquement à l'État lésé? Qu'en est-il des victimes individuelles? Est-ce que le DIH leur accorde un droit à la réparation?

En ce qui touche au droit coutumier applicable aux conflits armés internationaux, l'Étude du CICR sur le droit international humanitaire coutumier men-

le droit à la réparation : "Aucune disposition du présent Protocole relative à la responsabilité pénale des individus n'affecte la responsabilité des États en droit international, notamment l'obligation de réparation ".

28 Sylvie S. Junod, Claude Pilloud, Jean de Preux et Bruno Bruno Zimmermann, dans J. Pictet, dir., Commentaires des Protocoles additionnels du 8 juin 1977 aux Conventions de Genève de 1949, Genève, Comité International de la Croix-Rouge, 1986 à la p. 1079, para. 3645, en ligne : CICR < http://www.icrc.org/Web/fre/sitefre0.nsf/htmlall/p0421?OpenDocument > (date d'accès : $1^{\text {er }}$ décembre 2009) [ci-après Commentaires du CICR PI et PII].

29 Étude du CICR sur le DIH coutumier, supra note 25 à la p. 537: "A State responsible for violations of international humanitarian law is required to make full reparation for the loss or injury caused".

30 CGI, supra note 15, art. 51; CGII, supra note 15, art. 52; CGIII, supra note 15, art. 131; CGIV, supra note 15, art. 148: article commun aux quatre Conventions de Genève.

31 Commentaires du CICR CGIV, supra note 5 à la p. 645.

32 Projet d'articles sur la responsabilité de l'État, supra note 7, art. 31. 
tionne que la réparation peut être demandée par les États, mais aussi directement par les individus. Son obtention s'envisage toutefois par des mécanismes plus larges que ceux qui sont discutés ci-dessous, en application des articles $3 \mathrm{CIV}$ (1907) et 91 PI. Il est fait mention, par exemple, des ententes (interétatiques et autres) et des actes d'État unilatéraux par lesquels les victimes individuelles sont directement indemnisées. Il est vrai que ces mécanismes constituent une voie d'accès à la réparation, mais nous sommes d'avis que ces dispositifs présentent certaines lacunes pour les victimes individuelles. L'Étude du CICR sur le DIH coutumier confirme, cependant, que les articles 3 CIV (1907) et 91 PI précités n'indiquent pas qui est bénéficiaire de l'obligation de réparation et quels sont les mécanismes de révision des réclamations ${ }^{33}$. Si certains auteurs inferent de ces articles l'existence d'un droit individuel secondaire de demander réparation pour violations des « droits humanitaires » substantifs, la plupart d'entre eux soutiennent que ces règles régissent uniquement les relations entre États et que, quoiqu'elles puissent être appliquées au bénéfice des victimes, elles ne peuvent pas être invoquées directement par ces dernières. Qu'en est-il vraiment?

Des auteurs, tels que Rudolf Dozler, soutiennent l'impossibilité d'une application directe de l'article 3 CIV (1907), en affirmant que le droit de La Haye n'accorde aucun droit aux victimes qui ont subi un dommage imputable à un État résultant d'une violation du droit international. Il justifie sa position en citant entre autres Vattel, Brownlie ainsi que la synthèse de 1986 de la Loi américaine sur les relations étrangères ${ }^{34}$. D'autres auteurs, tels que A. W. Freeman ${ }^{35}$ et G.H. Aldrich $^{36}$, ont tenu des propos similaires. Pierre d'Argent considère lui aussi que le fait d'interpréter ces articles de façon à y voir un droit individuel à un recours et à une réparation sollicite très largement le texte, qu'elle n'est pas conforme à la volonté de leurs auteurs et qu'elle a été souvent, de toute façon, rejetée par la jurisprudence ${ }^{37}$. D'Argent ne voit pas dans ces articles un fondement suffisant pour établir un droit individuel qui serait transposable directement dans les ordres juridiques internes. Il ne ferme toutefois pas complètement la porte à une reconnaissance éventuelle de ce droit, admettant que :

il n'est pas impossible qu'à la suite de l'évolution du droit international des droits de l'homme, le droit des gens en

33 Étude CICR sur le DIH coutumier, supra note 25 à la p. 544.

34 Rudolf Dozler, "The Settlement of War-Related Claims :Does International Law Recognize a Victim's Private Right of Action? Lessons After 1945 » (2002) 20 Berkeley Journal International Law. 296 à la p. 308 (voir directement dans le texte de Dozler pour la référence à la loi américaine). Mentionnons au passage que Dolzer ne fait aucune allusion de l'article $91 \mathrm{PI}$.

35 A..Freeman, "Responsibility of the State for Unlawful Acts of Their Armed Forces " (1955) 88 Recueil des cours de l'Académie de droit international de La Haye 267 à la p. 333.

36 George H. Aldrich, "Individuals as Subjects of International Humanitarian Law » dans Jerzy Makarczyk, dir., Theory of International Law at the Threshold of the $21^{\text {st }}$ Century. Essays in Honour of Krzystof Skubiszewski, La Haye, Kluwer, 1996, 851 [ci-après Aldrich].

37 Pierre D’Argent, "Des règlements collectifs aux règlements individuels (collectivisés)? la question des réparations en cas de violations massives des droits de l'homme " (2003) 5 International Law FORUM du droit international 10 à la p. 13 [D'Argent]. Voir aussi du même auteur, Les réparations de guerre en droit international public. La responsabilité internationale des États à l'épreuve de la guerre, Bruxelles et Paris, Bruylantet LGDJ, 2002. 
vienne à consacrer au bénéfice des individus le droit d'obtenir réparation de leurs préjudices causés par certaines violations particulièrement graves du jus in bello lorsqu'elles emportent des atteintes à certains droits humains fondamentaux universellement reconnus et en tout temps indérogeables ${ }^{38}$.

Cette référence à l'évolution du droit à la réparation dans le temps et la pratique a aussi été utilisée dans les Commentaires du CICR CGIV, par rapport à l'article 148 (commun aux quatre Conventions de Genève) statuant sur l'impossibilité de s'exonérer de sa responsabilité étatique en cas d'infractions graves dont nous avons fait mention plus haut, pour en arriver, malgré tout, à des résultats différents. Notons que ces commentaires ont été formulés dans les années 1950, peu de temps après l'adoption des Conventions de Genève :

À notre avis, l'article 148 a pour objet d'empêcher que, dans une convention d'armistice ou dans un traité de paix, le vaincu ne soit contraint de renoncer à toute réparation due à raison d'infractions commises par des personnes se trouvant au service du vainqueur. En effet, en ce qui concerne la réparation matérielle des infractions à la Convention, il n'est pas possible, tout au moins en l'état actuel du droit, d'imaginer que les personnes lésées puissent intenter directement une action contre l'État au service duquel se trouvait l'auteur de l'infraction. Seul un État peut formuler de telles revendications à l'égard d'un autre État. Ces réclamations rentrent généralement dans le cadre de ce qu’on a appelé les «réparations de guerre». Il paraîtrait injuste que les individus fussent punis alors que l'État au nom de qui ils agissent - et parfois sur ses instructions - serait libéré de toute responsabilité [nos soulignements] ${ }^{39}$.

On peut voir dans cette analyse une infirmation de la théorie de l'application directe de l'article 3 CIV (1907) aux victimes individuelles. Cette interprétation de "l'état actuel du droit » dans les années 50 nous surprend, toutefois un peu, considérant l'histoire et le contexte de négociation ayant mené à l'adoption de l'article 3, au début du XXe siècle. En effet, comme le rapporte Frits Kalshoven ${ }^{40}$, la question de la réparation individuelle était prise en considération dans les textes normatifs dès 1899 : par exemple, les articles 52 et 53 de la Convention (II) concernant les lois et coutumes de la guerre sur terrét1 visaient l'indemnisa-

38 D’Argent, ibid. à la p. 14.

39 Commentaires du CICR CGIV, supra note 5 aux pp. 645 et 646.

40 Frits Kalshoven, "State Responsibility for Warlike Acts of the Armed Forces " (1991) 40 I.C.L.Q. 827 à la p. 830 [ci-après Kalshoven, "State Responsibility "].

41 Convention (II) de La Haye concernant les lois et coutumes de la guerre sur terre et son Annexe : Règlement concernant les lois et coutumes de la guerre sur terre, 29 juillet 1899, B.T.S. 1901/11, aux pp. 19-28, en ligne : CICR <http://www.icrc.org/dih.nsf/FULL/150?OpenDocument> (date d'accès : $1^{\text {er }}$ décembre 2009). 
tion à fournir aux communes et aux habitants qui sont sous occupation lorsque sont réclamées des réquisitions en nature ou de services pour les besoins de l'armée, actes qui sont, en soi, légaux. Kalshoven nous apprend ensuite que, lors la Deuxième Conférence internationale de la Paix de La Haye du 18 octobre 1907, le représentant allemand, major général Von Gündell, a proposé l'ajout de deux articles, le premier visant la compensation des personnes neutres et le second celle de la Partie adverse. Finalement, la Conférence a pris le parti de fusionner les deux articles en un seul, pour donner l'article 3 tel qu'on le connait ${ }^{42}$. Reste que le principe d'invocation de la responsabilité de l'État dans le but de permettre à la victime d'obtenir une compensation pour les dommages subis, reposant sur la proposition allemande, n'a jamais semblé être sujet de controverse lors de la Conférence. Ceci fait dire à Kalshoven que " [t] he article is unmistakably designed to enable [individual beneficiaries] to present their bills directly to the State, i.e. to its competent (military or other) authorities, either during or after war $»^{43}$ et que " $[t]$ he question of responsibility of one State vis-à-vis another State apparently never entered the minds of the draftsmen [of article 3] ${ }^{44}$. Il est vrai que certains auteurs n'ont pas une opinion aussi tranchée que Kalshoven sur l'histoire de l'adoption de l'article 3 CIV (1907). Par exemple, l'étude approfondie des Travaux préparatoires ayant mené à l'adoption de cet article réalisée par Micaela Frulli ${ }^{45}$ et reprise par Andrea Gattini ${ }^{46}$, nous apprend que cette disposition a donné lieu à certaines discussions. Frulli cite, en effet, une intervention d'un délégué britannique pour venir souligner les difficultés pratiques inhérentes à la reconnaissance d'un droit individuel qui découlerait de l'obligation de compensation monétaire qui incombe aux États :

Je ne conteste pas l'obligation qui existe pour une Puissance belligérante de dédommager ceux qui ont été victimes de la violation des lois et des coutumes de la guerre et la GrandeBretagne ne désire aucunement se soustraire à ses obligations. Je ferai seulement observer qu'il est souvent fort difficile de constater cette violation et la portée du dommage causé. Proclamer le principe est facile, mais il est bien difficile de l'appliquer sans soulever de contestations qui nuisent aux bons rapports des États qui doivent résoudre le problème ${ }^{47}$.

42 Voir Kalshoven, "State Responsibility ", supra note 41 à la p. 832, n. 20. Il est intéressant de noter que Kalshoven identifie les bénéficiaires de l'obligation posée par l'article 3 comme étant uniquement les ressortissants ennemis et les civils neutres, et non les combattants ou les prisonniers de guerre, même si certaines dispositions du Règlement à la Convention IV de 1907 visent le traitement qui est accordé à ces derniers.

43 Ibid. à la p. 836.

44 Ibid. à la p. 838.

45 Micaela Frulli, "When Are States Liable Toward Individuals for Serious Violations of Humanitarian Law? The Markovic Case » (2003) 1 Journal of International Criminal Justice 406 [ci-après Frulli, "When Are States Liable»].

46 Andrea Gattini, "To What Extend are States Immunity and Non-Justiciability Major Hurdles to Individual Claims for War Damages?" (2003) 1 Journal of International Criminal Justice 348 aux pp. 350 et s. [ci-après Gattini, «To What Extent »].

47 "Deuxième Conférence internationale de la Paix du 15 juin au 18 octobre 1907 ", dans, Actes et 
L'analyse des Travaux préparatoires fait dire à Frulli que l'introduction de cet article avait deux objectifs : premièrement, établir que chaque Puissance belligérante est responsable des violations des règles commises par ses forces armées et, deuxièmement, établir pour les États parties l'obligation d'indemniser les victimes civiles ressortissantes d'un État neutre ou de l'État ennemi comme étant le seul mode de réparation de ces violations. Ce constat lui fait affirmer, pour l'article visé, que « there is still ambiguity with respect to the right of individuals to directly seek and obtain compensation $"^{48}$. Nous verrons plus loin qu'outre les discussions durant les Travaux préparatoires de la CIV (1907), rien dans la jurisprudence subséquente et dans la pratique générale n'a laissé voir l'émergence d'un droit individuel d'obtenir directement réparation. Ceci explique donc un peu mieux la position adoptée par le CICR dans ses Commentaires au sujet des articles prévoyant l'impossibilité d'exonérer sa responsabilité étatique qui se trouvent dans les quatre Conventions de Genève.

Le constat n'est pas nécessairement plus clair en ce qui a trait à l'article $91 P I$ dans lequel la notion d'indemnité est aussi évoquée, sous une formulation presque identique à celle de l'article $3 C I V$ (1907). Elle vise non seulement les violations des dispositions du Protocole, mais aussi celles des quatre Conventions de Genève; le libellé est donc assez large. Les Travaux préparatoires ne nous fournissent cependant pas d'information cruciale sur le contexte d'adoption. Tel que nous le rapporte Frulli ${ }^{49}$, c'est un délégué vietnamien qui proposa l'article, en affirmant qu'il s'agissait là d'une volonté de réaffirmer le principe de réparation dont disposait déjà l'article 3. Le but recherché était certainement d'élargir le spectre d'application de la réparation, mais il n'est pas aussi clair de savoir si le délégué vietnamien, ainsi que tous les États présents, songeait à la situation d'une réparation individuelle. L'examen de la Conférence diplomatique ayant mené à l'adoption des deux Protocoles additionnels auquel Kalshoven a procédé nous donne toutefois un peu plus d'information. Il nous apprend en effet qu'on peut lire dans les Actes de la Conférence que les parrains de l'article 91 PI avaient puisé leur inspiration «de la destruction et des ravages qui ont résulté des guerres d'agression coloniale et néo-coloniale infligées sur le territoire de pays d'Asie où la population était faible et mal armée, tel qu'il était arrivé au Vietnam et dans certains pays africains " [notre traduction $]^{50}$. Reste que la question de l'existence ou non d'un droit individuel à la réparation n'a pas été soulevée lors du processus de rédaction de l'article 91 PI. Il est donc difficile de connaître, plus en détail, la portée qu'on a voulu conférer à l'article. Selon l'interprétation de Kalshoven, l'article 91 engage la responsabilité de la partie au conflit vis-à-vis d'un État ou, à la limite, une communauté ou un groupe mais non vis-à-vis d'une victime individuelle ${ }^{51}$. La lecture des Commentaires du CICR PI et PII au sujet de l'article 91 PI laisse entrevoir une autre possibilité ouvrant la porte sur la reconnaissance d'un droit individuel :

documents, La Haye, 1907, vol. I, à la p. 147, tel que cité dans Frulli, "When Are States Liable », supra note 46 à la p. 417 [Travaux préparatoires CGIV (1907)].

48 Ibid. à la p. 418.

49 Frulli, "When Are States Liable », supra note 46 à la p. 416.

50 Kalshoven, "State Responsibility" supra note 41 à la p. 845.

51 Voir par ex. ibid., supra note 41, à la p. 846. 
Les ayants droit à l'indemnité seront normalement des Parties aux conflits, ou leurs ressortissants. [...]

Sauf cas exceptionnels, les personnes de nationalité étrangère lésées par le comportement illicite d'une Partie au conflit devront s'adresser à leur propre gouvernement qui fera valoir leur plainte auprès de la ou des Parties auteurs de la violation, mais, on peut relever, depuis 1945 , une tendance à reconnaitre l'exercice de droits individuels [notes omises - notre soulignement $]^{52}$.

On peut s'étonner d'une telle affirmation de la part du CICR, surtout lorsqu'on la compare avec celle faite trente ans plus tôt au sujet de l'article 148 commun aux quatre Conventions de Genève, où le droit individuel à la réparation semblait infirmés s $^{3}$ Il est, en effet, difficile de réconcilier le tout. On peut toutefois voir dans ce dernier commentaire un obiter sur l'état du droit, et non sur la portée d'application de l'article 91 PI. En tout état de cause, nous croyons que ce sont ces Commentaires $d u$ CICR, à la lumière du contexte d'adoption de l'article 3 $C I V$ (1907), qui ont fait dire à Frulli:

I may thus be contented that Article 91 also lays down a rule binding on the contracting parties to the effect that they are mandated to pay damages caused to individuals by humanitarian law violations committed by their armed forces. It still remains unclear whether individuals have a corresponding right to seek compensation within domestic legal orders. ${ }^{54}$

Notons en outre qu'en 2005, la Commission d'enquête internationale sur le Darfour des Nations Unies s'est fondée sur les articles 3 CIV (1907) et 91 PI pour affirmer que les violations graves du droit humanitaire et des droits humains n'entraînent pas uniquement une responsabilité pénale des auteurs, mais aussi une responsabilité de l'État (ou la partie au conflit assimilable à l'État) au nom duquel l'acte a été commis. La Commission d'enquête a même spécifié que cette responsabilité impliquait le paiement d'une compensation à la victime, sans toutefois qu'il soit fait mention de l'existence d'un droit individuel à la compensation revenant directement à la victime $e^{55}$.

Il ne faut finalement pas omettre de parler de l'effort récent fourni par les Nations Unies dans la systématisation des règles émanant de différentes sources du droit international public pouvant bénéficier aux victimes à travers les Prin-

52 Commentaires du CICR PI et PII, supra note 29 à la p. 1082, au para. 3656.

53 Voir l'extrait des Commentaires du CICR CGIV, supra note 5 aux pp. 645 et 646.

54 Frulli, "When Are States Liable " supra note 46 à la p. 418.

55 Report of the International Commission of Inquiry on Darfur to the United Nations SecretaryGeneral pursuant to Security Council Resolution 1564, $1^{\text {er }}$ février 2005, Doc. NU S/2005/60 (2005) aux para. 593 et 597, en ligne : http://www.un.org/News/dh/sudan/com_inq_darfur. pdf (date d'accès : $1^{\text {er }}$ décembre 2009) : « [T] here has now emerged in international law a right of victims of serious human rights abuses (in particular, war crimes, crimes against humanity and genocide) to reparation (including compensation) for damage resulting from those abuses. ” 
cipes fondamentaux et directives concernant le droit à un recours et à réparation ${ }^{56}$. Puisqu'on n'y formule pas de nouvelles règles, la valeur d'un tel document ne réside pas dans son aspect novateur, mais bel et bien dans sa fonction de compilation des mécanismes de mise en ouvre des droits des victimes qui existent déjà au niveau normatif. Qui plus est, ce document a nécessité un travail d'une valeur inestimable de la part des États : celui de clarifier la teneur et la portée des obligations qu'ils se sont donnés. En effet, ces Principes doivent refléter les normes minimales du droit international ${ }^{57}$. L'existence même de ce document vient donc appuyer le constat que le système actuel évolue de façon à reconnaître, de plus en plus, le droit d'accès à la justice que possède les victimes individuelles de violations du DIH.

Ceci étant dit, l'analyse des articles 3 CIV (1907) et 91 PI à laquelle nous venons de procéder nous permet de constater que plusieurs interprétations s'offrent à nous et que la place que leur réserve le droit humanitaire n'est pas clairement définie dans les textes normatifs. À la lumière de la doctrine, nous sommes toutefois à l'aise d'affirmer, comme plusieurs autres auteurs l'ont fait avant nous ${ }^{58}$, que

56 Principes fondamentaux et directives concernant le droit à un recours et à réparation des victimes de violations flagrantes du droit international des droits de l'homme et de violations graves du droit international humanitaire, Rés. AG 60/147, Doc. Off. AG NU, 60 sess., supp. nº 49, Doc. NU A/RES/60/147 (2005): cette résolution de l'Assemblée générale des Nations Unies est le fruit de nombreuses années de travail. Le gouvernement chilien fut, tout d'abord, l'instigateur de ce projet, lorsqu'il débuta son processus de réparation des victimes au sein de l'État. Le besoin d'avoir entre les mains un outil pratique visant les droits des victimes, toutes sources confondues, s'est fait ressentir très clairement dans le déroulement de ces opérations de réparation. La même situation s'est présentée avec la Commission de vérité et réconciliation péruvienne. Voir Ipsita Mondal, "Second Consultative Meeting on «The Basic Principles and Guidelines on the Right to a Remedy and Reparation for Victims of Violations of International Human Rights and Humanitarian Law -20-23 October 2003, Geneva " International Movement Against All Forms of Discrimination and Racism (IMADR), en ligne : <http://www.imadr.org/old/ geneva/2003/2nd.CM.restitution.html> (date d'accès : $1^{\text {er }}$ août 2007).

57 Ibid. à l'annexe "General Observations ", au para. 11, à la p. 5.

58 Voir par ex. Éric David, Principes de droit des conflits armés, $2^{\mathrm{e}}$ éd., Bruxelles, Bruylant, 1999 au n ${ }^{\circ}$ 427, à la p. 570; Theodore Meron, Human Rights and Humanitarian Norms as Customary Law, Oxford, Oxford University Press, 1989 à la p. 224; Marco Sassòli, "State Responsibility for Violations of International Humanitarian Law " (2002) 84 Revue internationale de la Croix-Rouge 401 à la p. 419 [ci-après Sassòli, «State Responsability »] : « [...] in international humanitarian law [...] many rules are formulated in a human rights-like manner as entitlements of war victims. In such cases the only problem is procedural, i.e. that the injured individuals have no standing in the usual procedures for the settlement of disputes. Substantively they do have, however, an entitlement under international law " [note omise]. Voir aussi EmanuelaChiara Gillard, "Reparations for violations of international humanitarian law " (2003) 851 Revue internationale de la Croix-Rouge 529 à la p. 536 [ci-après Gillard, "Reparations »] : "While there is general consensus that there is no reason for limiting the right to compensation referred to in the Hague Convention and Additional Protocol I to States and that individual victims should also benefit, problems have arisen when such persons have attempted to enforce the right to reparations - usually compensation - directly before national courts " [note omise]; Zegveld, "Remedies for victims", supra note 23 à la p. 506 : «The liability of parties to a conflict to pay compensation for violations of IHL committed by persons forming part of their armed forces could entail an obligation to compensate not only States but also individual victims. [...] Several experts have taken the view that the very purpose of the article has been to confer rights directly on individuals " [notes omises]; Riccardo Pissillo Mazzeschi, "Reparation Claims by 
ces deux dispositions consacrent, dans une certaine mesure, le droit individuel des particuliers d'obtenir réparation des préjudices découlant des violations du droit humanitaire. Il demeure difficilement envisageable de trancher définitivement sur la question de savoir si la victime possède en DIH un droit procédural de présenter elle-même sa requête. Considérant la difficulté d'interprétation des articles, la question de leur application directe ou non sera traitée de manière plus approfondie par la suite, en s'attardant sur l'interprétation jurisprudentielle de ces dispositions.

Pour l'instant, nous pouvons affirmer que, lors de l'adoption du PI en 1977 et la reprise quasi intégrale du langage de l'article 3 CIV (1907) à l'article 91, l'intention des États était de s'affranchir - un tant soit peu — de la conception classique du droit international public et de reconnaitre la place de plus en plus définie qu'occupe l'individu dans ce milieu «étatique ». On reconnait toutefois du même souffle que l'intention n'allait peut-être pas aussi loin que de créer un véritable droit procédural pour les individus, engendrant ainsi une institution novatrice en droit international public. Il s'agit plutôt minimalement d'une reconnaissance de l'individu comme bénéficiaire des protections qu'offre le droit humanitaire et d'une prise en considération de ses intérêts. On peut appuyer cette interprétation en citant le rapporteur de la III commission de la Conférence diplomatique de Genève de 1949 qui a déclaré que :

Il ne suffit pas d'accorder les droits aux personnes et d'imposer aux États une responsabilité; il faut encore fournir aux personnes protégées les appuis qui leur sont nécessaires pour faire valoir leurs droits, sans quoi elles seraient juridiquement désarmées en face de la Puissance au pouvoir de laquelle elle se trouve $[\ldots]^{59}$.

Cette affirmation, qui est en quelque sorte une application de la maxime " a right without a remedy is not a right at all ", pourrait venir renforcer la position selon laquelle le droit humanitaire attribue un droit à la réparation aux victimes de violations. Il faut, malgré tout, replacer cette citation dans son contexte et comprendre que les " appuis nécessaires " consistent à obliger la Puissance au pouvoir à fournir aux personnes protégées les moyens nécessaires pour communiquer avec la Puissance protectrice, le CICR et les sociétés nationales. L'obligation ne semble donc pas nécessairement s'étendre jusqu'à fournir les moyens procéduraux de faire valoir un droit secondaire à la réparation dans l'éventualité d'une violation. Toutefois, ce type d'affirmation vient renforcer la position, qui est la nôtre, selon laquelle le droit des individus d'obtenir réparation au niveau international existe, malgré le fait qu'il semble improbable que l'individu puisse

Individuals for State Breaches of Humanitarian Law and Human Rights: An Overview » (2003) 1 Journal of International Criminal Justice 339 aux pp. 341 et 342 [ci-après Mazzeschi]: « In our opinion, Article 3 Hague Convention $n^{\circ}$ IV and Article 91 of Protocol I should therefore be jointly interpreted as rules providing for an obligation of reparation in favour both of states and of injured individuals".

59 Actes de la Conférence diplomatique de Genève de 1949, vol.II-A à la p. 805 tel que cités dans les Commentaires du CICR CGIV, supra note 5 à la p. 230, art. 30. 
exercer et exécuter directement ce droit à travers des mécanismes et recours internationaux. Si l'on peut affirmer qu'en droit international humanitaire, il y a eu un passage de l'étatique à l'individuel dans une certaine mesure, il est beaucoup plus difficile de soutenir qu'il y a eu passage d'individuel à individualisé, et qu'il est presque impossible d'argumenter un passage à la justiciabilité, compte tenu, nous le verrons plus loin, de l'état de la jurisprudence.

Finalement, il y a lieu de préciser ici que tout ce débat autour de la notion d'indemnisation des victimes en droit humanitaire conventionnel n'englobe que les conflits armés internationaux. Le fait que la notion de réparation ne fait pas partie du droit des traités s'appliquant aux conflits armés non internationaux est incontesté. Ceci est une lacune considérable du DIH, qui est toutefois atténuée par le fait que la Règle 150 de l'Étude du CICR sur le DIH coutumier dont nous avons discuté plus haut s'applique aux conflits armés internationaux et non internationaux, permettant ainsi de réclamer réparation contre les groupes d'opposition armés, en plus de l'État. Reste que, lorsque l'on sait que parmi les seize conflits armés majeurs répertoriés en $2008^{60}$, aucun n'est un conflit armé non international (une situation qui se répète depuis les cinq dernières années), on voit la portée limitée des discussions sur l'impact du droit conventionnel en ce qui a trait à la réparation.

\section{L'APPLICATION DIRECTE DU DROIT HUMANITAIRE EN DROIT INTERNE : EXAMEN DE JURISPRUDENCES NATIONALES : LES RECOURS CONTRE L'ÉTAT ${ }^{61}$}

Pour évaluer la justiciabilité du droit à la réparation prévu par le DIH par rapport à la victime, un survol de l'état de la doctrine était nécessaire; un constat de l'état de la jurisprudence se fondant sur les articles 3 CIV (1907) et 91 PI est tout aussi fondamental. Le précédent judiciaire pèse lourd dans la balance quand vient le temps d'évaluer la justiciabilité d'un droit. En effet, l'alinéa 31(3)c) de la Convention de Vienne sur le droit des traités du 23 mai $1969^{62}$ portant sur les règles générales d'interprétation indique qu'il "sera tenu en compte, en même temps

60 Lotta Harbom et Peter Wallensteen, « Major Armed Conflicts » dans Stockholm International Peace Research Institute (SIPRI) dir., Yearbook 2008 Armement, Disarmement and International Security, Stockholm, 2006, annexe 2A, en ligne : <http://www.sipri.org/yearbook/2009/02/02A> ( date d'accès: $1^{\text {er }}$ décembre 2009).

61 À noter que lorsque qu'une norme émanant d'un traité de droit international public octroie directement un recours, la qualification dudit traité comme étant ou non directement applicable en droit interne ("self executing treaty") est primordiale. Même s'il ne s'agit pas en soi d'une question du droit interne de l'État où l'action est entamée, il est important de s'y attarder pour évaluer comment sont mis en œuvre les droits qui peuvent être interprétés comme étant justiciables individuellement. Nous évoquerons donc ce concept fréquemment dans les prochaines pages, puisqu'il constitue en quelque sorte, le pont entre le droit international humanitaire et le droit interne. Voir, à ce sujet, Christopher Greenwood, " Rights to compensation of former prisoners of war and civilian internees under article 3 of Hague Convention n IV, 1907 : Expert Opinion » dans H. Fijita, I. Suziki et K. Nagano, dir., War and the Right of Individuals: Renaissance of Individual Compensation, Tokyo, Nippon Hyoron-sha, 1999, 59 à la p. 68.

621155 R.T.N.U. 354. 
que le contexte $[\ldots]$ de toute pratique ultérieurement suivie dans l'application du traité par laquelle est établi l'accord des parties à l'égard de l'interprétation du traité ». Donc, pour pouvoir mieux interpréter le droit conventionnel, il est des plus pertinents d'examiner la pratique des États à travers les décisions qui émanent de leurs propres tribunaux.

Nous avons recensé certaines affaires dans lesquelles des victimes individuelles de violations de droit international humanitaire ont tenté d'obtenir réparation de la part de l'État auquel était imputable la violation. Nous verrons à la lumière d'une analyse empirique ${ }^{63}$ de la pratique des États que les tribunaux nationaux rejettent dans presque tous les cas les actions individuelles contre les États qui se fondent directement sur des dispositions du droit humanitaire. Puisque la pratique judiciaire se veut généralement uniforme au sein d'un même État, nous procéderons à une analyse jurisprudentielle ordonnée par pays dans lequel l'action civile a été entamée.

\section{A. Les États-Unis : les affaires Tel-Oren et Princz}

La question à laquelle nous tenterons de répondre dans la présente section est de savoir, en application du raisonnement posé dans l'affaire Fosterv. Neilson ${ }^{64}$, si le droit à la réparation dont dispose la $C I V(1907)$ et le PI peut être mis en œuvre par les tribunaux américains sans qưil soit nécessaire que le Congrès légifere à ce sujet.

L'Affaire Tel-Oren ${ }^{65}$ paraît, à première vue, éloignée de l'analyse que nous effectuons du droit individuel à la réparation pour les victimes de violations du DIH. En effet, les demandeurs, majoritairement des citoyens israéliens, poursuivent la Libye, mais aussi d'autres entités non étatiques, telles que l' Organisation de Libération de la Palestine (OLP) et le Congrès palestinien d'Amérique du Nord, pour les dommages subis à la suite d'un attentat armé sur un autobus, en mars 1978. Or, la Cour, aussi bien en première instance qu'en appel, ne semble pas avoir effectué la qualification préliminaire de la situation pour savoir s'il s'agit de troubles internes ou d'un conflit armé régi par le DIH. Nous pouvons donc difficilement expliquer pourquoi la Cour d'appel du district de Columbia, sous la plume du Juge Edwards, s'est prononcée sur la non-applicabilité directe en droit interne de l'article 3 CIV (1907). Nous analyserons tout de même ses commentaires, en prenant soin de les classer dans la catégorie des obiter dictum.

L'Affaire Tel-Oren donne le ton de l'interprétation constante qu'ont adoptée

63 Il ne s'agit pas d'une revue jurisprudentielle in extenso. Les décisions qui sont examinées ci-après ont été sélectionnées parce qu'elles émanent toutes de juridictions nationales, qu'elles ont toutes été intentées par une ou plusieurs victimes individuelles contre un État et qu'elles se fondent ou qu'elles analysent les articles 3 CIV (1907) et/ou $91 \mathrm{PI}$.

64 Foster v. Neilson, 27 U.S. (2 Pet.) 253, 314 (1829): « Our constitution declares a treaty to be the law of the land. It is, consequently, to be regarded in courts of justice as equivalent to an act of the legislature, whenever it operates of itself, without the aid of any legislative provision. But when the terms of the stipulation import a contract when either of the parties engages to perform a particular act, the treaty addresses itself to the political, not the judicial, department. "

65 Tel Oren v. Libyan Arab Republic, 517 F. Supp. 542 (D.D.C. 1981); 726 F.2d 774 (D.C. Cir. 1984) [ci-après Affaire Tel Oren]. 
les tribunaux américains par rapport à l'applicabilité directe en droit interne du droit à la réparation tel que prévu dans la CIV(1907) durant les dernières décennies. Voici le passage pertinent de la décision:

The Hague Conventions similarly cannot be construed to afford individuals the right to judicial enforcement. Although the Conventions contain no language calling for implementing legislation, they have never been regarded as law private parties could enforce.

La Cour poursuit en expliquant plus en détail les implications - politiques et diplomatiques - qu'elle entrevoit dans l'éventualité où l'article 3 serait interprété comme attribuant un droit individuel à la réparation :

If they were so regarded, the code of behavior the Conventions set out could create perhaps hundreds of thousands or millions of lawsuits by the many individuals, including prisoners of war, who might think their rights under the Hague Conventions violated in the course of any large-scale war. Those lawsuits might be far beyond the capacity of any legal system to resolve at all, much less accurately and fairly; and the courts of a victorious nation might well be less hospitable to such suits against that nation or the members of its armed forces than the courts of a defeated nation might, perforce, have to be. Finally, the prospect of innumerable private suits at the end of a war might be an obstacle to the negotiation of peace and the resumption of normal relations between nations. It is for these reasons that the Conventions are best regarded as addressed to the interests and honor of belligerent nations, not as raising the threat of judicially awarded damages at war's end. The Hague Conventions are not selfexecuting. ${ }^{66}$

La même interprétation des Conventions de La Haye a été adoptée, moins d'une décennie plus tard. L'Affaire Goldstar Panama ${ }^{67}$ a été introduite devant les tribunaux américains au début des années 90 par un regroupement d'entreprises panaméennes contre l'État américain pour obtenir réparation des dommages subis à la suite des émeutes et des rafles ayant fait rage dans le sillage de l'invasion du Panama. Les demandeurs ont argué que les États-Unis avaient une obligation d'assurer la sécurité des civils sous leur contrôle. Bien que cette affaire implique des personnes morales, elle constitue un élément important de l'établissement de la pratique des États subséquente à l'adoption de l'article 3 CIV (1907), puisque c'est sur cette disposition que les demandeurs-appelants fondent leur action en

66 Ibid. (D.C. Cir. 1984) à la p. 810.

67 Goldstar (Panama) S.A. v. United States, 763 F. Supp. 1154 (D.D.C. 1991); 967 F.2d 965 (4th Cir. 1992) [ ci-après Affaire Goldstar Panama]. 
dommages et intérêts. La position adoptée en première instance puis confirmée par la Cour d'appel américaine dans cette affaire, a l'avantage d'être sans équivoque : l'article 3 n'étant pas applicable directement en droit interne américain, il ne peut pas fonder une demande individuelle en réparation. Cette position s'appuie sur une application stricte de la théorie du " self executing treaty » voulant qu'une convention internationale ne puisse attribuer un droit d'action individuel que si elle en dispose de façon expresse, comme l'indique cet extrait souvent cité de la décision :

Courts will only find a treaty to be self-executing if the document, as a whole, evidences an intent to provide a private right of action. ${ }^{68}$

Cette interprétation de l'article 3 est à nouveau maintenue dans l'Affaire Prin$c z^{69}$, à la différence que, cette fois-ci, la Cour a infirmé la décision de première instance. En effet, M. Hugo Princz, un citoyen américain ayant survécu aux camps de concentration nazis lors de la Seconde Guerre mondiale, se tourna vers la justice américaine et y intenta un recours en réparation contre l'État allemand se fondant sur l'article $3 C I V$ (1907), n'ayant pas été en mesure d'obtenir compensation de la part de l'Allemagne à travers les mécanismes traditionnels de protection diplomatique en raison de sa citoyenneté américaine. La District Court avait, tout d'abord, mis de côté l'application de la doctrine de l'immunité de l'État et avait reconnu sa compétence d'entendre la demande de compensation présentée par Princz. La Cour de première instance semblait toutefois des plus mal à l'aise face à l'application de la théorie de l'immunité étatique pour empêcher la compensation monétaire d'une personne ayant subi des «actes barbares $»^{70}$. La décision fut, cependant, infirmée en appel lorsque l'argument de l'immunité étatique fut accepté, et ce, malgré une forte dissidence du Juge Wald. Le tribunal, pour justifier sa décision, cite expressément les affaires Goldstar et Tel-Oren:

[...] Mr. Princz argues, «the compensation provisions of the Hague Convention are in conflict with the [Federal Sovereign Immunity Act']s immunity provision, thus invoking its exception for prior existing treaty obligations.» Binding precedent is clearly to the contrary, and clearly correct.

68 Ibid. (4th Cir. 1992), au para. 968.

69 Princz v. Federal Republic of Germany, 813 F. Supp. 22 (D.D.C. 1992); 26 F.3d 1166 (D.C. Cir. 1994) [ci-après Affaire Princz].

70 Voir ibid. (D.D.C. 1992), au para. 26 : "This Court finds that the Foreign Sovereign Immunity Act has no role to play where the claims alleged involve undisputed acts of barbarism committed by a one-time outlaw nation which demonstrated callous disrespect for the humanity of an American citizen, simply because he was Jewish. [...] What this Court holds today is that under the circumstances of this case, a nation that does not respect the civil and human rights of an American citizen is barred from invoking United States law to block the citizen in his effort to vindicate his rights ". 
[...] The cases are unanimous, however, in holding that nothing in the Hague Convention "even impliedly grants individuals the right to seek damages for violation of [its] provisions." Tel-Oren v. Libyan Arab Republic [...]; accord Goldstar (Panama) S.A. v. United States [...]

For the foregoing reasons, we conclude that none of the exceptions to sovereign immunity provided in the FSIA applies to the facts alleged by Mr. Princz ${ }^{71}$.

À la lumière de ces décisions, on peut affirmer que l'interprétation que les tribunaux américains donnent à l'article 3 CIV (1907) comme n'ayant pas d'application directe en droit interne, est assez uniforme. En ce qui a trait aux Conventions de Genève dans leur ensemble, il y a certaines raisons de croire qu'elles auraient une applicabilité directe en droit interne. Il existe, en effet, un certain courant jurisprudentiel selon lequel les droits individuels prévus à la CGIII (relative aux prisonniers de guerre) s'appliqueraient directement en droit interne ${ }^{72}$. Reste que, tel qu'il a été déclaré dans United States $v$. Postal ${ }^{3}$, la doctrine du « self-executing treaty " reste une notion très difficile à cerner qui pose de nombreux problèmes. Il nous est donc difficile d'adopter une position ferme et arrêtée à ce sujet dans le cadre du présent article, le contexte américain étant aussi bien particulier. En ce sens, l'impact de l'utilisation contemporaine du Alien Tort Claims Act ${ }^{74}$ et du Torture Victim Protection Act of $1991^{75}$ permettant un élargissement en matière de compétence des tribunaux américains pour des violations du droit international public a changé le paysage judiciaire du pays. Toutefois, cet élément ne sera pas abordé ici, faute d'espace ${ }^{76}$. Reste que l'examen de la jurisprudence, tout au

71 Ibid. (D.C. Cir. 1994), au para. 1175. Il est fort intéressant de voir comment cette affaire s'est poursuivie à l'extérieur de l'arène judiciaire. Et pour cause, la Fondation allemande Remembrance responsibility and future fut fondée en 2000 dans la mouvance de l'Affaire Princz dans le but de rendre la compensation financière accessible aux victimes de travaux forcés et d'autres injustices commises à l'époque du régime national-socialiste : voir généralement en ligne : http://www. stiftung-evz.de/eng/> (date d'accès : $1^{\text {er }}$ décembre 2009).

72 Voir United States v. Noriega, 808 F.Supp. 791, 799 (S.D. Fla. 1992) qui a tranché que la CGIII était applicable directement en droit américain (" [I] $\mathrm{t}$ is inconsistent with both the language and spirit of [the GPW] and with our professed support of its purpose to find that the rights established therein cannot be enforced by individual POW in a court of law "), ce qui permettait au Général Noriega de déposer une poursuite aux États-Unis pour trancher sur les violations alléguées, suite à sa détention dans une prison américaine. Il a, en outre, été affirmé dans cette affaire que la doctrine du self-execution était complexe et pas particulièrement bien comprise (para. 797). Voir aussi Padilla ex rel. Newman v. Bush, 233 F. Supp. 2d 564, 590 (S.D.N.Y. 2002) et United States v. Lindh 212 F. Supp. 2d 541, 553-554 (E.D. Va. 2002), dans lesquelles il a été affirmé que la CGIII avait force de loi sous la "Supremacy Clause " que l'on retrouve à l'article VI, para. 2 de la Constitution américaine.

73589 F.2d 862, 876 (5th Cir. 1979) : « the self-execution doctrine [is] one of the most confounding in treaty law».

7428 U.S.C. $\$ 1350$ [ci-après ATCA].

75 Pub. L. No. 102-256, 106 Stat. 73 (1992) [ci-après TVPA].

76 Pour des analyses plus approfondies de l'impact du ATCA et du TVPA sur la sanction des violations des droits humains par les tribunaux américains, voir Beth Stephens, "Individuals 
moins, celle relative à l'article $3 C I V$ (1907), ferme quand même la porte quant à l'exercice d'un droit individuel à la réparation pour les victimes de violations de DIH devant les tribunaux américains.

\section{B. Le Japon : les Sengo Hosho Saiban}

"Sengo Hosho Saiban" est le terme japonais utilisé pour définir les réclamations individuelles en réparation post bellum présentées au cours des trente dernières années devant les tribunaux japonais contre l'État japonais en rapport aux actes posés par celui-ci lors de la Seconde Guerre mondiale (qu'il s'agisse d'enrôlement forcé dans les forces armés, d'esclavage sexuel, de traitements infligés lors de la détention ou de déplacement forcé). Il est à noter que, dans la plupart des affaires, les demandeurs étaient d'origine japonaise, mais aussi coréenne, taïwanaise, chinoise, philippine, indonésienne et malaisienne ${ }^{77}$.

Dans le traitement de ces demandes, le Japon semble avoir opté, comme l'ont fait et le font encore les États-Unis, pour une interprétation restrictive de la doctrine du "self-executing treaty», ne voyant dans les conventions de droit international un droit individuel à un recours que lorsqu'il est explicitement mentionné :

The general position of Japanese courts on the matter is that individuals cannot be regarded as having the right to demand compensation from an offending State for violations of inter-

Enforcing International Law: The Comparative and Historical Context " (2002) 52 DePaul Law Review 433 "Translating Filartiga: A Comparative and International Law Analysis of Domestic Remedies For International Human Rights Violations " (2002) 27 Yale J Int'l L 1 [ci-après Stephens "Translating "]; " Taking Pride in International Human Rights Litigation » (2001) 2 Chicago Journal of International Law 485; William S. Dodge, "The Constitutionality of the Alien Tort Statute: Some Observations on Text and Context " (2002) 42 Va. J. Int'l L. 687; George Norris Stavis, "Collecting Judgements in Human Rights Tort Cases - Flexibility for Non-Profit Litigators?» (1999) 31 Colum. Hum. Rts. L. Rev. 209 aux pp. 211-219 [ci-après Stavis].

77 Pour une analyse poussée sur la jurisprudence japonaise au sujet de la compensation des victimes de guerre, voir Shin Hae Bong, "The Right of War Crimes Victims to Compensation Before National Courts - Compensation for Victims of Wartime Atrocities - Recent Developments in Japan's Case Law " (2005) 3 Journal of International Criminal Justice 187 [ci-après Hae Bong] et Sayuri Umeda "Japan: WWII POW \& Forced Labor Compensation Cases " (sept. 2008), en ligne : United States Law Library of Congress http://www.loc.gov/law/help/japan-wwiipow.pdf (date d'accès : 7 décembre 2009) [ci-après Umeda]. Voir aussi Tetsuo Ito "Japan's Settlement of Post World-War II Reparations and Claims " et et Karen Parker et Jennifer F. Chew "Reparation: A Legal Analysis " dans Roy L. Brooks (dir.), When sorry isn't enough: the controversies over apologies and reparation for human injustices, New York, New York University Press, 1999 [ci-après When sorry isn't enough]; Barry A. Fisher, "Japan's Postwar Compensation Litigation» (2000) 22 Whittier L. Rev. 35. Les jugements plus récents sont accessibles dans leur langue originale en ligne : Courts of Japan http://www.courts.go.jp/.. En outre, un résumé des poursuites contre l'État japonais entamées par les victimes de violences sexuelles avant et durant la Seconde Guerre mondiale (en date du 3 décembre 2004) est disponible en ligne : Violence Against Women in War - Network Japan « Lawsuits against the Government of Japan filed by the survivors in Japanese Courts " http://www1.jca.apc.org/vaww-net-japan/english/ sexualslavery/courtcase.html (date d'accès : 7 décembre 2009) [ci-après VAWW-NET Japan, «Court Cases »]. 
national law unless such a procedure is explicitly provided for in a treaty, and that the interpretation of scholars invoked by the plaintiffs can neither be confirmed nor has been supported by State practice ${ }^{78}$.

En ce qui a trait, plus spécifiquement, à l'application directe en droit interne de l'article 3 CIV(1907), on peut citer une décision dans laquelle les tribunaux japonais ont reconnu, à la lumière des Travaux préparatoires qui ont mené à l'adoption dudit article $3^{79}$, que ce dernier avait été conçu pour «soulager " les individus qui ont souffert lors d'un conflit armé, mais qu'un droit individuel à un recours contre l'État fautif ne peut en être déduit, tel qu'en témoigne cette traduction non-officielle du dispositif du jugement :

[TRADUCTION]

1. In general, public international law regulates interstate rights and obligations;

2. When a treaty is applied to individual claims against the state in a domestic court, the contents of the claim under the provision of the treaty should be very clear from the viewpoint of balance of powers and stability of law;

3. The text of Hague Convention IV never suggested that an individual had claims against the state;

4. The drafting record of the Convention did not support the plaintiffs' view, but rather was based on the assumption that compensation for individuals would be provided through pursuing diplomatic protection by the state to which the individual belonged. ${ }^{80}$

La même interprétation de l'article $3 C I V$ (1907) a été retenue par les tribunaux japonais dans l'Affaire du massacre de Nanking ${ }^{81}$ introduite devant les tri-

78 Hae Bong, Ibid. à la p. 189.

79 Travaux préparatoires CGIV (1907), supra note 48.

80 Voir Dutch POW Case, 1685 HANREI JIHÕ 19, 29-32 (Tokyo Dist. Ct. Oct. Nov. 30, 1998) tel que cité dans Umeda, supra note 78, aux pp. 16 et 17; voir aussi U.K. POWs Case, 1685 HANREI JIHÕ 4, 13-18 (Tokyo Dist. Ct. Nov. 26, 1998). Pour ces deux affaires, les décisions furent maintenues en appel pour les mêmes motifs : Dutch POWs Case, 1769 HANREI JIHÓ 61 (Tokyo High Ct., Oct. 11, 2001) et U.K. POWs Case, 1802 HANREI JIHŌ 76 (Tokyo High Ct., Mar. 27, 2002) mais la Cour suprême a refusé d'entendre l'appel en 2004. Voir aussi Chinese victims of sexual violence c. Japan, 1823 HANREI JIHŌ (Tokyo D. Ct., 2003) 6182 (non-applicabilité directe du droit à la réparation prévu par l'article 3 CIV (1907) en droit interne), tels que cités dans Hae Bong, supra note 78.

81 Chinese victims of Nanking Massacre and inhuman acts by the Unit 731 of the Japanese army c. Japan, Tokyo D. Ct., 22 septembre 1999, tel que cité dans Hae Bong, supra note 78 [ci-après Affaire du massacre de Nanking]. Voir aussi les commentaires de Inokuchi Hiromitsu et Nozaki Yoshiko, "Court Cases, Citizen Groups, and the Unresolved Issues of War: Updates and Brief Commentary "; en ligne: Center for Research and Documentation on Japan's War Responsibility http://space.geocities.jp/japanwarres/center/english/Courtcas.htm) (date d'accès : 7 décembre 2009) [ci-après Hiromitsu et Yoshiko]. 
bunaux japonais durant les années 90 . Les demandeurs dans cette affaire étaient les victimes chinoises ainsi que les ayants cause des victimes décédées des suites de violations du droit humanitaires commises lors de l'invasion de Nankin par les troupes japonaises en $1937^{82}$. Ils ont fondé leur recours sur l'article $3 \mathrm{CIV}$ (1907). La Cour a refusé de voir dans cette disposition un droit au recours individuel. Elle a argumenté au surplus que l'obtention de réparation pour les victimes peut « réveiller d'anciens démons » et contrecarrer les efforts de prévention d'un nouveau conflit :

To admit the rights of individuals to claim, from foreign States, compensation for damages suffered during a past war without relying upon diplomatic negotiations between States is, viewed from the broader framework to maintain peace and security between States and nations and in each region, as a whole, equivalent to leaving seeds of conflict, and harmful in view of preventing future wars, even though it is compatible with justice for the individual in the sense of general civil law. ${ }^{83}$

Ce raisonnement soulève des questions fondamentales qui outrepassent un cadre strictement juridique et auxquelles nous ne tenterons pas de répondre ici. On peut néanmoins souligner que, quoiqu'un argument se fondant sur le maintien de la paix durable entre les peuples peut venir entraver l'accès d'une victime à la réparation, il y a un minimum de mauvaise foi dans le fait d'affirmer que la réparation compensatoire individuelle peut devenir une cause potentielle d'un nouveau conflit armé international : justice et réconciliation ne se rejoignent pas toujours, mais rien n'empêche que les deux principes puissent suivre des chemins parallèles et complémentaires. Il est, en outre, intéressant de rapporter ici que le Women's International War Crimes Tribunal, une instance quasi-judiciaire émanant de la société civile et ayant un pouvoir de recommandation ${ }^{84}$, n’a pas suivi

82 Plusieurs violations du DIH ont été commises par les Forces armées japonaises sur la population civile lors de cette attaque : on estime à plus de 200000 le nombre de Chinois tués et à environ 20000 le nombre de femmes violées. Des traitements inhumains et cruels ont été réalisés par l'Unité 731 chargée de recherche bactériologique. Voir notamment : Masahiro Yamamoto, Nanking: anatomy of an atrocity, Praeger, 2000; Joshua A. Fogel, The Nanjing Massacre in history and historiography, University of California Press, 2000; Iris Chang "The Nanking Massacre ", dans When sorry isn't enough, supra note 78.

83 Affaire du massacre de Nanking, supra note 82. Voir aussi la traduction d'un autre passage du jugement qu'offre le Center for Research and Documentation on Japan's War Responsibility: "Considering the justice of the whole human race, the problems of compensation with respect to former enemy nations should be solved comprehensively by the postwar friendship treaty between the nations, even if it is the case that each individual's justice cannot be restored in terms of civic law. $[D]$ enying individual rights to claim compensation for damage (in the case of war) is reasonable, if we presume that avoiding another war is the supreme cause.» : Hiromitsu et Yoshiko, supra note 82.

84 Voir, en ligne: Violence Against Women in War - Network Japan, «The Women's International War Crimes Tribunal on Japan's Military Sexual Slavery " http://www1.jca.apc.org/vaww-netjapan/english/womenstribunal2000/whatstribunal.html (date d'accès : 7 décembre 2009). Au sujet du pouvoir de recommandation, en ligne : Violence Against Women in War - Network 
ce courant jurisprudentiel ; il a en effet conclu que l'article 3 CIV (1907) confere un droit individuel à la compensation ${ }^{85}$.

Soulignons finalement que d'autres obstacles se sont dressés sur le chemin des victimes de violations du DIH commises par l'État japonais vers le prétoire : un premier obstacle est celui de la " doctrine de l'irresponsabilité de l'État ", qui veut que ce dernier ne puisse jamais être tenu responsable pour les actes juri imperii (ce qui inclut tous les actes posés par ses forces armées). Cette doctrine, très proche parente de la doctrine d'immunité étatique, existait dans le système juridique japonais d'avant-guerre et a souvent été invoquée par les tribunaux pour refuser d'allouer une compensation aux victimes de violations du DIH, et ce, jusqu'à récemment ${ }^{86}$. Ce postulat de droit interne, dont la compatibilité même avec la notion d'État de droit est sujette à caution a ,toutefois, été mis de côté dans plusieurs décisions ${ }^{87}$.

Un deuxième obstacle réside dans l'application de la théorie de la renonciation étatique aux « réparations de guerre " dans un traité de paix bilatéral comme impliquant une renonciation pour toutes les victimes individuelles. Quoiqu'il a été conclu dans l'affaire Shimoda ${ }^{88}$ que l'effet des traités de paix ne se faisait sentir qu'au niveau interétatique, l'argument fut soulevé à plusieurs reprises... avec un succès mitigé. Par exemple, dans l'affaire Shimonosek ${ }^{89}$, le Tribunal de première instance, ayant reconnu que le traitement infligé aux «femmes de réconfort "

Japan, "Charter of The Women's International War Crimes Tribunal On Japan’s Military Sexual Slavery ", art. 14 (Incorporating Modifications Agreed upon During the Hague Meeting, 2627 October 2000) http://www1.jca.apc.org/vaww-net-japan/english/womenstribunal2000/ charter.html> (date d'accès : 7 décembre 2009).

85 Prosecutors and the People of the Asia-Pacific region c. Japan (2000) Affaire $\mathrm{n}^{\circ}$ PT-2000-1-T (Women's International War Crimes for the Trial of Japan Military Sexual Slavery), au para. 1053, en ligne : Violence Against Women in War - Network Japan http://www1.jca.apc. org/vaww-net-japan/english/womenstribunal2000/Judgement.pdf) (date d'accès : 7 décembre 2009) : «[...] we find that article 3 of the 1907 Hague Regulations was intended to protect the right of the victimized persons and those who are permitted to claim on their behalf to seek compensation as individual."

86 Voir par ex. deux affaires dans lesquelles la doctrine de la non-responsabilité de l'État a été retenue : Philippine "comfort women» c. Japan, Tokyo D.C., 9 octobre 1998 et Residents of HongKong c. Japan, Tokyo D.C., 16 June 1999, tels que cités dans Hae Bong, supra note 78.

87 Voir par ex. Chinese victims of forced labour c. Japan and the company A (non divulgué), Kyoto D. C., 15 January 2003; Chinese victims of forced labour c. Japan and Hazamagumi Inc., Tokyo D.C., 11 mars 2003 (non publié); Chinese victims offorced labourc. Japan and Rinko Corporation, Niigata D.C., 26 mars 2004, tels que cités dans Hae Bong, supra note 78.

$88: \lll[\ldots]$ an individual's claim in international law is not recognized until it is provided for by a treaty and the right of bringing action and other procedural guarantees by which the individual can assert the claim have come into existence internationally." : Shimoda c. The State, [1964], 32 I.L.R. 626 (Jap., Tokyo D.C.); en ligne : CICR, « National Case law » http://www.icrc.org/ ihl-nat.nsf/46707c419d6bdfa24125673e00508145/aa559087dbcf1af5c1256a1c0029f14d?O penDocument, au para. 5, au sous-para 5 (date d'accès : 7 décembre 2009).

89 Cette affaire est aussi connue sous le nom de "Kankama moto ianfu Sosho" (Kankama Former Comfort Women Case), 1642 HANREI JIH 24, 28-30 (Yamaguchi Dist. Ct., Shimonoseki Branch April 27, 1998), tel que cité dans Umeda, supra note 78, $\mathrm{n}^{\circ} 18$. Voir aussi Etsuro Totsuka, "Translations: Commentary on a Victory for "Comfort Women" : Japan's Judicial Recognition of Military Sexual Slavery" (1999) 8 Pac. Rim L. \& Pol'y J. 47.

90 Pour une proposition de définition: en ligne, Asian Women's Fund, "Who were the Comfort Women?-Who were the Comfort Women?», http://www.awf.or.jp/e1/facts-00.html (date 
était extrêmement inhumain et constituait une pratique abominable, a accordé la somme symbolique de 300000 yens (approximativement 2,700 dollars US) à chacune des trois demanderesses pour compenser les dommages moraux qu'elles avaient subis. Ce jugement fut cependant infirmé en appel, sur le fondement que toutes les réclamations en compensation avaient été réglées dans les traités de paix $^{91}$.

\section{La Grèce : l'Affaire de la préfecture de Voiotia}

La série d'ententes interétatiques conclues entre l'Allemagne et les pays alliés laisse à penser que les questions de réclamations relatives à la Seconde Guerre mondiale contre l'Allemagne étaient désormais exclues ${ }^{92}$. Il semble toutefois que ces renonciations de la part d'un État floué n'empêchent pas les individus de présenter une action civile en dommages devant un tribunal national ${ }^{93}$, tel que semble le démontrer cette illustration grecque.

Cinquante ans après l'attaque du 10 juin 1944 du village de Distomo touchant la Préfecture de Voiotia en Grèce, 259 descendants et ayants cause des victimes ont intenté une action civile devant les tribunaux grecs contre l'État allemand. Cette cause est maintenant connue sous le nom de l'Affaire de la Préfecture de

d'accès : 7 décembre 2009) : "The so-called "wartime comfort women" were those who were taken to former Japanese military installations, such as comfort stations, for a certain period during wartime and forced to provide sexual services to officers and soldiers. Authors who wrote about these women in the postwar Japan called them "jugun ianfu (comfort women joining the army)". And when the Japanese government first faced the issue of these women, it adopted this term, "jugun ianfu," and the AWF, when it started in 1995, it used this term as well. But in historical wartime documents we only find the term "ianfu (comfort women)". Therefore, we now always use this term "ianfu (comfort women)". "

91 Korean "comfort women» c. Japan, Hiroshima High Court, 29 mars 2001, tel que cité dans Hae Bong, supra note 78. En ce qui a trait à la réaction de la société civile à l'égard de ce jugement, voir par ex. Okinawa Peace Network of Los Angeles (OPN-LA), «Los Angeles Organizations Protest Legacy of Japanese Militarism: Groups Angered by Japanese High Court Decision on "Comfort Women" and Textbook Controversy", en ligne : http://uchinanchu.com/about comfort_women_press_release.htm (date d'accès : 7 décembre 2009); "Comfort women payout overturned "AFP - The Straits Times (30 mars 2001), en ligne : <http://www.hartford-hwp. com/archives/55/537.html>; "Japan overturns sex slave ruling " BBC News (29 mars 2001) en ligne : ‘http://news.bbc.co.uk/2/hi/asia-pacific/1249236.stm) (date d'accès : 7 décembre 2009).

92 Il y a tout d'abord eu en 1953 l'Accord de Londres sur la dette externe de l'Allemagne dans lequel 33 États se sont entendus pour suspendre toutes les réclamations en lien avec la guerre jusqu'à ce qu'il y ait entente définitive sur la question : voir London Agreement on German External Debts, 17 février 1953, 333 R.T.N.U. 3, au para. 5(2). Il avait, toutefois, été entendu entre les États parties que le moratoire ne visait pas les indemnités à verser aux victimes des persécutions nazies. Cette clause d'exclusion du moratoire est connue sous le nom d' "Israël Exception " et se trouve à l'Annexe VIII de l'Accord. C'est dans cette foulée que fut signé, en 1956, un accord bilatéral entre l'Allemagne et la Grèce attribuant à ce dernier un montant de 115 millions deutsche marks. Le Traité portant règlement définitif concernant l'Allemagne du 12 septembre 1990, mieux connu sous le nom du Traité de Moscou «2+4» ne parle aucunement des réclamations, ce qui sous-entend que les questions des réclamations contre l'Allemagne étaient désormais exclues. Pour plus de détails à ce sujet : Gattini, "To What Extent ", supra note 47 à la p. 356.

93 Voir Gattini, «To What Extent », supra note 47 à la p. 359. 
Voiotia $^{94}$. En première instance, l'Allemagne a décidé de ne pas comparaître. Le 30 octobre 1997, un jugement contre l'Allemagne fut rendu, lui ordonnant de verser la somme de 5 millions deutsche marks aux ayants cause des victimes du massacre de Distomo. Le gouvernement allemand décida d'aller en appel devant la Cour suprême grecque, l'Areios Pagos, et d'invoquer l'argument de l'immunité diplomatique. Contre toute attente, la plus haute instance du pays a rejeté, le 5 mai 2000, l'appel de l'Allemagne, considérant qu'étaient en cause des violations de normes de jus cogens (contenues dans le droit humanitaire) commises par ses forces armées plus d'un demi-siècle auparavant. Les deux instances grecques ont appliqué les articles 3 CIV (1907) et 46 des Règlements de La Haye et ont tranché en faveur des demandeurs, les autorisant à soumettre directement leur réclamation pour compensation ${ }^{95}$.

Cette affaire constitue un moment-charnière dans la justiciabilité des droits individuels à la réparation dans le contexte de conflits armés. Le vent a toutefois tourné lorsque les demandeurs, forts de leurs deux victoires et jugement en main, se sont vus refuser l'autorisation du Ministère de la Justice grec requise par l'article 923 du Code de procédure civile grec pour effectuer une saisie par rétention ("attachement ") d'institutions allemandes se trouvant en territoire grec $^{96}$, en vue d'exécuter le jugement. La requête en exécution fut donc déférée à la Cour suprême grecque. Il y a eu revirement de situation : elle refusa, cette fois-ci, de mettre en œuvre le jugement, affirmant que la saisie de propriétés pouvait légitimement être refusée afin de maintenir des " relations amicales " entre les deux États. Il est important de souligner qu'il n’a pas été question ici de ne pas reconnaître un droit individuel à la réparation dans l'article 3 CIV (1907). Pour les besoins de notre analyse, même si les demandeurs n'ont pu obtenir réparation, ils n'ont pas essuyé un refus sur la base que leur droit à la réparation n'existait pas, mais bien parce que les tribunaux grecs ont jugé qu'il ne serait pas souhaitable que ce droit soit exercé contre des biens particuliers, par risque de mise en péril des relations interétatiques. En outre, nous devons indiquer que la décision finale d'empêcher, en pratique, l'exécution du jugement, a reposé également sur les épaules de l'exécutif et du judiciaire, chacun ayant adopté une position relevant plutôt de la conservation de bonnes relations diplomatiques que de la mise en ouvre du droit à la réparation.

Mentionnons, en dernier lieu, que cette affaire a aussi eu des suites en Allemagne. En effet, après l'échec ultime de refus d'exécution du jugement rendu par les instances judiciaires grecques, les ayants cause des victimes du massacre de Distomo se sont retournés vers les tribunaux allemands pour obtenir réparation. La Bundesgerichtshof (BGH) a rendu sa décision, l'Affaire Distomo ${ }^{97}$, en 2003

94 Loriginal de ce jugement est rapportée dans Nomiko Vima (1998) 246 ff., tel que cité dans Gattini, "To What Extent ", supra note 47 à la p. 359, n 10. À noter que le terme "Affaire Distomo " réfere à l'action qui fut introduite subséquemment devant les tribunaux allemands.

95 Pour une analyse plus poussée, voir Gattini, "To What Extent », supra note 47 à la p. 359 et s. Voir aussi l'Étude du CICR sur le DIH coutumier, supra note 25 à la p. 545.

96 Nommément : l'Institut Goethe, l'Institut allemand d'archéologie et deux écoles allemandes à Athènes et à Saloniki : Gattini, "To What Extent ", supra note 47 à la p. 360.

97 BGH, décision du 26 juin 2003, III ZR 245/98, publiée dans NJW 2003 à la p. 3488 et s., tel que rapportée dans : Sabine Pittorof, «Compensation Claims for Human Rights Breaches 
dans laquelle elle rejetait l'action présentée par les citoyens grecs. Elle a adopté une position conservatrice à l'égard de l'existence d'un droit individuel d'obtenir réparation, se replaçant au moment où la violation avait été commise, soit lors de la Seconde Guerre mondiale. La BGH a affirmé que la notion de compensation dans le contexte d'un conflit armé impliquait uniquement une relation entre États et que les individus n'y avaient pas leur place. Elle s'est rabattue sur la théorie de la protection diplomatique pour indiquer que c'était le seul mécanisme accessible aux victimes leur permettant d'obtenir réparation. Nous pouvons, néanmoins, nuancer la position adoptée par la Cour fédérale allemande en mettant l'emphase sur le fait qu'elle a considéré l'état du droit au moment de la violation, sans opter pour une approche évolutive. Elle n’a donc pas fermé la porte aux réclamations individuelles ayant pris naissance plus récemment, ne s'étant pas prononcée sur l'état actuel du droit à la réparation individuelle dans un contexte post bellum.

\section{L'Italie : l'Affaire Markovic ${ }^{88}$}

Jamais une instance judiciaire, qu'elle soit internationale ou nationale, n'a entendu au fond une affaire dans laquelle les victimes civiles (ou les États au bénéfice des victimes) d'opérations militaires réalisées par l'OTAN dans le cadre du conflit en ex-Yougoslavie en 1999 ont demandé réparation ${ }^{99}$. Les tribunaux

Committed by German Armed Forces Abroad During the Second World War: Federal Court of Justice Hands Down Decision in the Distomo Case " (2004) 1 German Law Journal 5, en ligne : German Law Journal <http://www.germanlawjournal.com/article.php?id=359\#_edn3> (date d'accès : 1er décembre 2009). Voir aussi l'Étude du CICR sur le DIH coutumier, supra note 25 aux pp. 544 et 545.

98 Ordinanza della Corte di Cassazione, Seszioni Unite Civili, 8 février 2002, tel que cité dans N. Ronzitti, "Azioni belliche risarcimento del danno " (2002) 85 Rivista di diritto internazionale 682, tel que rapporté dans Mazzeschi, supra note 59, à la p. 345. Pour un résumé détaillé et une analyse approfondie de cette affaire, voir Frulli, "When are States Liable », supra note 46.

99 En effet, la Cour internationale de Justice (CIJ) a décliné compétence dans les poursuites entamées par la Serbie-Monténégro contre des États membres de l'OTAN alléguant l'usage illicite de la force et la commission de violations du droit humanitaire face aux populations civiles : voir les Affaires relatives à la licéité de l'usage de la force (Yougoslavie c. Belgique, Canada, France, Italie, Pays-Bas, Portugal et le Royaume-Uni), Objections préliminaires, [2004] C.I.J. rec. 1307. Les deux affaires contre les États-Unis et l'Espagne (Affaire relative à la licéité de l'usage de la force (Yougoslavie c. Etats-Unis et Espagne), Ordonnance du 2 juin 1999, [1999] C.I.J. rec. 916) ont été rejetées pour manque manifeste de compétence. La Cour Européenne des Droits de l'Homme a aussi déclaré irrecevable la requête alléguant que le bombardement de la Radio-Télévision serbe par l'OTAN a violé la Convention européenne de sauvegarde des Droits de l'Homme et des Libertés Fondamentales, 4 novembre 1950, 213 R.T.N.U. 221. N'ayant pas été persuadé de l'existence d'un lien juridictionnel entre les victimes et les États défendeurs, la Cour a décliné compétence : Bankovic c. Belgique (2001), 41 I.L.M. 517 (Cour Européenne des Droits de l'Homme). D'autres tentatives, à l'échelon national, ont échoué de la même façon; voir, par ex. l'Affaire Varvarin ( $1^{\mathrm{er}}$ nov. 2006) : rejet de l'action d'un groupe de citoyens serbes demandant compensation à l'Allemagne pour les attaques aériennes de l'OTAN ayant tué 10 civils et blessé 30 personnes; voir Reuters AlertNet, " German Court Reject Serbs Claim for NATO Attacks "(2 nov. 2006), en ligne : 〈http://www.alertnet.org/thenews/newsdesk/ L02720300.htm (date d'accès : 7 décembre 2009). Même en matière pénale, le Bureau du Procureur du Tribunal pénal pour l'ex-Yougoslavie a refusé d'ouvrir une enquête sur deux attaques lancées par les forces de l'OTAN : voir Final Report to the Prosecutor by the Committee Established to Review the NATO Bombing Campaign Against the Federal Republic of Yugoslavia, en 
italiens ne font pas exception. Lorsque des citoyens de l'ex-Yougoslavie ont entamé une action civile contre l'Italie pour les dommages subis lors de l'attaque de l'OTAN sur la tour de diffusion de la Radio Televizje Srbiijre [STR ${ }^{100}$, le plus haut tribunal du pays a conclu à une absence de compétence pour entendre l'affaire et a refusé de l'entendre au fond. En se fondant sur l'article $91 P{ }^{101}$, les demandeurs réclamaient le versement d'une indemnité monétaire pour compenser les dommages subis à la suite de violations alléguées du $\mathrm{DIH}^{102}$.

La Cour suprême italienne s'est retenue de se prononcer explicitement sur l'existence d'une obligation conventionnelle de verser compensation ou de tout droit corollaire permettant à la victime de demander cette compensation et n'a pas fait reposer sa décision de décliner compétence sur des arguments assimilables à une application de la doctrine du "self-executing treaty ». Elle fonda plutôt sa décision sur la théorie de l'« Acte de gouvernement ${ }^{103}$. Cette théorie, qui permet l'exercice discrétionnaire de la puissance publique, est un mécanisme de retenue judiciaire au bénéfice du gouvernement, lorsque celui-ci doit agir de façon à protéger les intérêts constitutionnels et politiques de la nation. L'utilisation de cette théorie ne fait pas l'unanimité dans la doctrine, tel que le démontre Frulli ${ }^{104}$.

\section{E. Conclusion sur l'analyse des recours individuels en réparation}

Par l'étude de cette série de décisions d'ordre civil émanant d'organes judiciaires nationaux qui impliquent une partie individuelle demanderesse réclamant compensation à un État en fondant une action sur le droit humanitaire conventionnel, nous avons constaté que la non-applicabilité directe du DIH conven-

ligne: Nations Unies, Tribunal pénal pour l'ex-Yougoslavie <http://www.icty.org/x/file/About/ OTP/otp_report_nato_bombing_en.pdf> (date d'accès : $1^{\text {er }}$ décembre 2009).

100 La responsabilité de l'État italien était invoquée par le fait qu'il a été impliqué dans le processus décisionnel et l'identification de cibles à atteindre au sein de l'OTAN et par le fait que des bases militaires italiennes ont été utilisées pour mener à terme les bombardements. Il est intéressant de noter, même si cela n'a pas été abordé par les tribunaux italiens puisque la cause n’a pas été entendue au fond, que la responsabilité étatique est alléguée malgré le fait qu’il était impossible d'identifier à quelle nationalité les avions appartenaient, lors des bombardements du 23 avril 1999.

101 Voir Frulli, "When are States Liable ", supra note 46 à la p. 409.

102 PI, supra note 16 , art. 35, 48, 51 et 79.

103 Comme l'indique Frulli, "When Are States Liable ", supra note 46 à la p. 410, cette théorie est connue sous le nom de " teoria dell'atto di governo" en Italie, " actes de gouvernement " en France, "Royal Prerogative " au Royaume-Uni et " Political Question Doctrine " aux ÉtatsUnis.

$104 \mathrm{Ibid}$. aux pp. 409 et s. : puisque l'étude de cette doctrine n'est pas l'objet du présent texte, nous ne prendrons pas position, à cet effet, et nous vous renvoyons à l'article de M. Frulli pour prendre plus amplement connaissance des débats entourant l'interprétation de la théorie d'acte de gouvernement à laquelle a procédé la Cour suprême dans l'Affaire Markovic. Voir aussi Institut de Droit international, Session de Milan - 1993, L'activité du juge interne et les relations internationales de l'Etat, (Neuvième Commission, Rapporteur : M. Benedetto Conforti), en ligne : Institut de Droit international <http://www.idi-iil.org/idiF/navig_chron1993.html> (date d'accès : $1^{\text {er }}$ décembre 2009). L'article 2 de cette résolution vient poser le principe que, si les tribunaux d'un État sont dans l'impossibilité d'arrêter un conflit armé, il leur est quand même permis de se prononcer sur les dommages subis par les individus, si les dommages sont les résultats d'une violation de règles de droit international public. 
tionnel et les diverses déclinaisons de la théorie de l'immunité diplomatique constituent les obstacles majeurs aux actions individuelles en réparation contre l'État. Nous devons donc nous rendre à l'évidence : si l'interprétation selon laquelle le droit humanitaire a évolué pour accorder des droits individuels justiciables aux victimes pour qu'elles obtiennent réparation peut très bien se défendre à la lumière de l'interprétation des textes habilitants, force est de constater que cette position n’a presque jamais passé le seuil des institutions judiciaires. Plus exactement, peu de tribunaux se sont mouillés à répondre à cet argument, ce qui laisse la voie encore libre pour de plus amples spéculations. Les faits démontrent toutefois, qu'il est très rare qu'un tribunal de droit interne accepte les réclamations individuelles en réparation contre un État fautif.

\section{UN MÉCANISME INTERNATIONAL DE PLAINTE ACCESSIBLE AUX VICTIMES DE VIOLATIONS DU DIH?}

En matière de mise en oeuvre, les dispositifs traditionnels prévus par le droit international public (DIP), comme la résolution pacifique des différends ou les moyens de pression réactifs, trouvent difficilement leur place dans le contexte d'un conflit armé. De plus, contrairement aux mécanismes prévus par le système de protection des droits humains, le DIH conventionnel ne prévoit pas de comité, commission ou tribunal qui soit compétent pour recevoir des plaintes individuelles, déterminer de façon indépendante si une violation a eu lieu et octroyer une réparation. Comme l'affirment Sassòli et Bouvier, «le DIH a dû trancher un des axiomes de la société internationale traditionnelle et prévoir des mesures d'exécution dirigées contre les individus qui violent ce droit, et pas uniquement contre les États responsables de ces violations ${ }^{105}$. Ces mesures d'exécutions individuelles peuvent être pénales ou civiles, les deux types se complétant et même se recoupant parfois. D'un côté, l'expansion du droit pénal international au cours des dernières années contribue à accroître l'effectivité du DIH. Par contre, l'autre mécanisme, celui de la sanction civile, ne connaît pas nécessairement un essor similaire. C'est dans cet l'esprit que nous évoquons ici brièvement le projet de création d'un "organe de supervision " (Supervisory Organ) international ayant pour fonction de traiter des réclamations individuelles présentées par des victimes de violations du droit humanitaire, en nous questionnant sur l'opportunité et la possibilité de créer un tel organe.

Cette idée a été développée par la Amsterdam International Law Clinic ${ }^{106}$, dans le sillage de l'Appel de La Haye pour la paix et la justice au XXIe siècle en mai

105 Sassòli Bouvier supra note 3, à la p. 271.

106 Pour une présentation détaillée du projet, voir Jann K. Kleffner, «Establishing an Individual Complaints Procedure for Violations of International Humanitarian Law ", en ligne : Universiteit van Amsterdam, Faculty of Law, Amsterdam International Law Clinic $<$ http://www 1.jur.uva.nl/ailc/Individual\%20 complaints $\% 20$ and $\% 20$ international $\% 20$ humanitarian\%20law.pdf> (date d'accès : $1^{\text {er }}$ décembre 2009) et, du même auteur, «Improving Compliance with International Humanitarian Law Through the Establishment of an Individual Complaints Procedure " (2002) 15 Leiden Journal of International Law 237. Voir aussi Jann Kleffner et Liesbeth Zegveld, "Complaints Procedure for Violations of International Humanitarian Law » (2000) 3 Y.B. Int'l Human. L. 384. 
$1999^{107}$. Premièrement, il est intéressant de noter qu'en ce qui a trait à la compétence ratione materiae, le spectre des types de violations donne lieu à un éventail de réclamations très large et englobe tous les textes normatifs applicables aux conflits armés ainsi que le droit coutumier. Il est des plus pertinent de souligner qu'il est considéré, dans ce projet de traité, que le DIH, par le truchement des articles $3 C I V$ (1907) et $91 \mathrm{PI}$, attribue aux individus des droits humanitaires justiciables. Il est toutefois admis par les rédacteurs du projet que la pratique des tribunaux nationaux ne va pas dans ce sens et qu'il est presque impossible pour les victimes d'exercer réellement leurs droits humanitaires. Face à cette limite, une autre approche proposée quant à la conceptualisation du fondement juridique du recours a été de passer par la notion de dommage ( injury »). L'idée serait pour l'organe de supervision d'avoir compétence pour entendre toutes les affaires où une violation d'une norme de DIH définie a causé un dommage à la victime-demanderesse, se rapprochant ainsi des concepts de base du régime de responsabilité civile (faute - dommage - lien de causalité).

Au sujet de la compétence ratione personarum (la compétence sur les personnes), le débat se situe plus autour de la notion de groupes armés non étatiques ${ }^{108}$ qu'autour de l'auteur individuel de la violation. La question "Contre qui la victime peut exercer son recours? " vise donc à examiner les problèmes reliés aux entités non étatiques (leur caractère temporaire, la difficulté d'établir la chaîne de commandement, etc.) et non aux agresseurs eux-mêmes. L'organe de supervision adjugerait donc contre les « parties aux conflits » que celles-ci soient un État ou un groupe armé non étatique.

En outre, les différentes formes que prendrait la réparation attribuée par l'organe de supervision seraient la restitution, la compensation, la réhabilitation et la satisfaction. Par contre, puisque les demandes pourraient très bien dépasser les capacités de l'organe de supervision, les auteurs du projet insistent sur le fait qu'il faut établir des mécanismes de réparation crédibles plutôt que de miner son autorité en prévoyant des moyens illusoires, sans toutefois que cette institution devienne un lieu de recommandations non contraignantes et de jugements déclaratoires "sans dents ". Pour tenter de ne pas cibler uniquement l'aspect monétaire de la réparation, le projet fait preuve d'originalité : par exemple, l'organe de supervision pourrait ordonner de mettre en place des mesures de réhabilitation médicales et psychologiques dans le dessein de restaurer la dignité des victimes. Dans le projet de traité établissant un organe de supervision quant aux violations du DIH, la compensation pécuniaire n'est donc pas un passage obligé vers la réparation.

La mise sur pied d'un tel organe est une perspective novatrice, certes, mais pas irréalisable au niveau strictement juridique, tout au moins. Le projet de traité

107 L'Agenda de La Haye pour la paix et la justice a été adopté par l'Assemblée générale des Nations Unies, tel qu'en témoigne la résolution A/54/98 du 20 mai 1999 (lettre datée du 17 mai 1999, adressée au Secrétaire général par le Représentant permanent du Bangladesh auprès de l'Organisation des Nations Unies), en ligne : <www.haguepeace.org> (date d'accès : $1^{\text {er }}$ décembre 2009), recommandation 13.

108 Les critères applicables pour qualifier un "groupe d'opposition armée " au sens du DIH sont établis dans la CGIII, art. 4, puis précisés dans Procureur c. Tadi (1995) Affaire no IT-94-1AR72 (Tribunal Pénal pour l'ex-Yougoslavie, Chambre d'appel), au para. 70 et s. 
identifie avec beaucoup de rigueur et de clairvoyance les différents éléments de droit qui doivent être pris en considération et organisés de façon cohérente dans le contexte d'un mécanisme individuel visant les violations du DIH. Il ne peut toutefois fournir aucune solution au problème que peut poser la réticence des États à créer ce nouvel organe. Si un nombre insuffisant d'États sont intéressés, si trop de réserves sont émises, si le consensus ne peut être atteint sur certains points, l'organe de supervision ne pourra exister. Toutefois, lorsqu'on se place du côté de la victime, il est évident que la création d'un tel mécanisme serait bénéfique. Considérant que les options des victimes sont déjà très réduites, que plusieurs d'entre elles contiennent leur part d'arbitraire et que la voie vers le prétoire est semée d'embûches, la création d'une nouvelle avenue n'est pas une initiative futile et inutile. Nous ne pouvons, néanmoins, pas ignorer la valeur probante des objections formulées par les tribunaux nationaux examinées ci-dessus (qu'il y aurait, par exemple, un grand nombre de demandes ou encore que ces demandes pourraient nuire aux relations entre les États impliqués) qui sont également valables pour un organisme international.

En outre, certains auteurs ne voient pas la nécessité de créer de nouvelles institutions internationales. Certains considèrent les voies de recours internationales, régionales mais surtout nationales qui existent déjà ${ }^{109}$ sont suffisantes. Il est vrai qu'un système judiciaire fonctionnel et éprouvé existe déjà au sein de la majorité des États et qu'il faut, tout au moins, s'attarder à examiner dans quelle mesure ce système peut, mais aussi doit, être utilisé pour permettre aux victimes de violations du DIH d'obtenir réparation. Nous affirmons par contre du même souffle que l'utilisation des tribunaux nationaux n'empêche a priori pas le développement d'un projet de mécanisme permanent de traitement des réclamations en réparation des victimes de violations du droit humanitaire. Il s'agit là d'une complémentarité fonctionnelle entre les différents mécanismes, chacun présentant ses limites et ses avantages propres.

Cela étant dit, nous devons préciser ici que l'idée de la mise sur pied d'une instance permanente n'a pas été priorisée par le Comité " Reparation for victims of armed conflicts » de l'International Law Association, ce qui constitue en soi un argument majeur contre l'instauration de celle-ci. En effet, lors de la Conférence de Toronto de 2008, les membres de ce comité ont préféré investir leurs efforts dans la rédaction d'une ébauche de statut-type pour des commissions internationales de compensation ad hoc:

109 Beth van Schaak, "In Defense of Civil Redress: The Domestic Enforcement of Human Rights Norms in the Context of the Proposed Hague Judgments Convention " (2001) 42 Harv.Int'lL.J. 141 à la p. 165. Voir aussi William J. Aceves, «Liberalism and International Legal Scholarship: The Pinochet Case and the Move Toward a Universal System of Transnational Law Litigation " (2000) 41 Harv.Int'l L.J. 129 à la p. 132; Menno T. Kamminga, "Towards a Permanent International Claims Commission for Victims of Violations of International Humanitarian Law », en ligne : Université de Maastricht <arno.unimaas.nl/show.cgi?fid=3610> (date d'accès : $1^{\text {er }}$ décembre 2009): «From a trial attorney's point of view, an individual complaints procedure may be more appealing than the compensation mechanism [... ]. While an individual complaints procedure might also be empowered, like the ICC, to award compensation it is difficult to imagine how these institutions could ever deal with the massive numbers of claims typically associated with an armed conflict ». 
It was also felt that it would not be feasible to favour the future establishment of a single, permanent compensation commission but rather to support the concept of ad hoc compensation commissions. It was, however, proposed to explore the advantages (and disadvantages) of the creation of a permanent body (a kind of permanent secretariat) the experienced members of which could assist in the process of establishing future ad hoc compensation commissions empowered to deal with claims resulting from violations of international law committed during specific armed conflicts. ${ }^{110}$

Cela nous fait donc dire que, malgré le grand potentiel existant avec une instance permanente, il est, de moins en moins, probable qu'elle ne voit jamais le jour. Reste que tout au moins, il est intéressant et pertinent de présenter le potentiel théorique d'une telle initiative, si ce n'est que pour jeter un éclairage probant sur l'état actuel et potentiel des mécanismes de mise en œuvre du DIH.

\section{CONCLUSION}

Aux termes de cette brève incursion dans le $\mathrm{DIH}$, nous sommes en mesure d'affirmer que l'existence d'un droit individuel à la réparation dans le cadre de conflits armés internationaux semble se lire dans deux dispositions du droit conventionnel (art. 3 CIV (1907) et 91 PI), quoique cela ne fasse pas l'unanimité dans la doctrine. Par contre, le courant jurisprudentiel, lui, tend fortement vers la non-applicabilité directe du DIH en droit interne. Face à ce constat, il serait pertinent de se pencher sur les autres mécanismes accessibles aux victimes dans leur quête de réparation pour les dommages subis dans le cadre d'un conflit armé : ceux prévus par le droit pénal international, les recours « classiques » du droit

110 International Law Association, "Committees ", Conference Report Toronto 2006, au para. 13 à la p. 3, en ligne : http://www.ila-hq.org/en/committees/index.cfm/cid/1018, (date d'accès : 7 décembre 2009). Exemples d'initiatives ad hoc de compensation des victimes de conflits armés : la Commission d'indemnisation des Nations Unies (CINU) pour l'Irak et le Koweït (United Nations Security Council Resolution 687 (Concerning the Restoration of Peace and Security in Iraq and Kuwait), Rés. CS 687 (1991) Doc. Off. CS NU, Doc. S/RES/687 (1991) : 2.7 millions de réclamations et près de 352.5 milliards de dollars en compensation. Pour plus d'informations, en ligne (en anglais) : <http://www.worldpress.org/specials/iraq/unscr687.htm> (date d'accès : $1^{\text {er }}$ décembre 2009); Agreement between the Government of the Federal Democratic Republic of Ethiopia and the Government of the State of Eritrea 40 International Legal Materials (2001) à la p. 260: plus de 400000 réclamations. Voir généralement, en ligne : <http://www.pca-cpa.org/ showpage.asp?pag_id=1215> (date d'accès : $1^{\text {er }}$ décembre 2009); Iran - United States Claims Tribunal, en ligne : <http://www.iusct.org> (date d'accès : $1^{\text {er }}$ décembre 2009); Commission for Looted Art in Europe (CLAE) (un organisme à but non lucratif indépendant dont le mandat est de représenter le European Council of Jewish Communities (ECJC) et la Conference of European Rabbis (CER)). Voir généralement, en ligne : <www.lootedartcommission.com> (date d'accès : $1^{\text {er }}$ décembre 2009); Housing and Property Claims Commission au Kosovo (Regulation $n^{\circ} 1999 / 23$ on the Establishment of the Housing and Property Directorate and the Housing and Property Claims Commission, UNMIK/REG/1999/23, 15 November 1999, en ligne : United Nations Mission in Kosovo <http://www.unmikonline.org/regulations/1999/reg23-99.htm> (date d'accès : $1^{\text {er }}$ décembre 2009). 
international public général (par exemple, la protection diplomatique, le régime de responsabilité étatique pour fait internationalement illicite et les mécanismes de résolution de conflits et de réparation ad hoc), le régime de protection du droit international public des droits de la personne et même les recours de nature interne impliquant le droit national, tels que des actions en torts ou en responsabilité civile. L'envergure de ce travail serait, toutefois, colossale et l'espace accordé ici ne nous permet pas de vous faire part de cette partie de la recherche. Nous pouvons, malgré tout, indiquer que la conclusion finale tendrait vers le fait qu'il n'existe pas une solution unique qui permet à toutes les victimes de violations du $\mathrm{DIH}$ d'accéder à la réparation. Chacun des mécanismes possède ses avantages et ses inconvénients, et nous ne croyons pas qu'il est possible - ni même opportun - d'identifier une " formule unique " qui permet d'obtenir réparation contre des entités auxquelles les violations sont imputables. En guise de conclusion, nous désirons porter l'attention du lecteur sur une autre piste de réflexion, qui fut déjà évoquée dans la doctrine, dans le contexte canadien ${ }^{111}$ : Devrions-nous militer pour des changements législatifs nationaux en droit privé pour une meilleure protection judiciaire des victimes de violations du droit international humanitaire? Plutôt que de créer de nouvelles instances internationales, ne devrait-on pas utiliser les structures déjà existantes et éprouvées et permettre aux tribunaux civils nationaux d'entendre les victimes sur leurs réclamations en réparation? Il s'agit, selon nous, d'une piste des plus intéressantes. Qui plus est, les États-Unis, avec l' ATCA, tracent la voie à suivre et permettent de voir dans cette possibilité de changement législatif une option bénéfique pour les victimes.

Il est clair qu'à ce jour, la voix des victimes de violations du DIH ne se fait pas entendre clairement et que leur accès au prétoire est parsemé d'embûches qui leur sont souvent fatales. Bien peu de personnes obtiennent des jugements en réparation, et encore moins réussissent à voir ces jugements se transposer dans la réalité. L'appréciation de cette question de réparation individuelle pour compenser les personnes qui subissent les conséquences les plus atroces des violations des lois de la guerre ne doit toutefois pas se faire au niveau quantitatif : même si uniquement un petit nombre de personnes réussissent à traverser les obstacles et engager la responsabilité de ceux qui ont violé leur droit de ne pas subir les affres de la guerre, il s'agit là d'une grande victoire en soi. L'être humain qui souffre peut ne jamais voir ses besoins de vengeance, de pardon, ou d'oubli assouvis, mais il ne devrait jamais se voir nier un accès à la justice lui permettant de demander d'être remis dans l'état où il était avant qu'il subisse les conséquences de la violation d'une règle de droit. C'est à lui, ensuite, de voir s'il désire exercer ce droit qui est inhérent à sa dignité humaine.

111 Caroline Davidson, "Tort au canadien: A Proposal for Canadian Tort Legislation on Gross Violations of International Human Rights and Humanitarian Law » (2005) 38 Vand. J. Transnat'l. L. 1403. 
